Published in final edited form as:

Nat Struct Mol Biol. 2016 May ; 23(5): 378-386. doi:10.1038/nsmb.3196.

\title{
Structural basis for therapeutic inhibition of complement C5
}

\author{
Matthijs M. Jore ${ }^{1}$, Steven Johnson ${ }^{1}$, Devon Sheppard ${ }^{1}$, Natalie M. Barber ${ }^{1}$, Yang I. Li ${ }^{2,3}$, \\ Miles A. Nunn ${ }^{4,5}$, Hans Elmlund ${ }^{6,7}$, and Susan M. Lea ${ }^{1,6,7}$ \\ ${ }^{1}$ Sir William Dunn School of Pathology, University of Oxford, Oxford, United Kingdom \\ ${ }^{2}$ MRC Functional Genomics Unit, Department of Physiology, Anatomy and Genetics, University of \\ Oxford, Oxford, United Kingdom \\ ${ }^{4}$ Centre for Ecology and Hydrology, Wallingford, United Kingdom \\ ${ }^{6}$ Department of Biochemistry and Molecular Biology, Biomedicine Discovery Institute, Monash \\ University, Melbourne, Victoria, Australia \\ ${ }^{7}$ Australian Research Council Centre of Excellence in Advanced Molecular Imaging, Monash \\ University, Melbourne, Victoria, Australia
}

\begin{abstract}
Activation of complement C5 generates the potent anaphylatoxin C5a and leads to pathogen lysis, inflammation and cell damage. The therapeutic significance of $\mathrm{C} 5$ inhibition is demonstrated by Eculizumab, one of the world's most expensive drugs. However, the mechanism of C5 activation by $\mathrm{C} 5$-convertases remains elusive and limits development of therapeutics. Here, we identify and characterize a novel protein family of tick-derived C5 inhibitors. Structures of C5 in complex with the novel inhibitors, the Phase I \& II inhibitor OmCI, and an Eculizumab Fab reveal three distinct binding sites on $\mathrm{C} 5$ that all prevent activation of $\mathrm{C} 5$. The inhibitor binding sites, and ability of all three C5-inhibitor complexes to competitively inhibit the C5-convertase, conflict with earlier steric inhibition models, suggesting a priming event is needed for activation.
\end{abstract}

For Correspondence: hans.elmlund@monash.edu or susan.lea@path.ox.ac.uk.

${ }_{5}^{3}$ Current address: Departments of Genetics and Biology, Stanford University, Stanford, United States of America.

${ }^{5}$ Current address: Akari Therapeutics Plc, London, United Kingdom

Author Contributions.

S.M.L. \& M.A.N. initially conceived the work. S.M.L. \& M.M.J. designed the biochemical experiments and M.M.J. performed them with the assistance of N.M.B. M.M.J. crystallized the complexes and the structures were solved by S.M.L. \& S.J. M.M.J., S.M.L., S.J. analyzed the structures. D.S. designed, collected and solved the NMR structure. H.E. designed the EM experiments and together with S.M.L. collected and analyzed the data. Y.I.L. processed the HiSeq data and assembled the transcriptome. All authors were involved in discussion of results and preparation of the manuscript.

Competing financial interests

M.M.J., M.A.N \& S.M.L. hold IP in the area of tick complement inhibitors.

Accession Codes

Structure factors and coordinates for X-ray structures have been deposited in the Protein Data Bank (PDB) with accession codes 5HCC, 5HCD \& 5HCE. Coordinates for the NMR structure have also been deposited in the PDB with accession code 5IEC and experimental restraints have been deposited in the BMRB (30024). The EM volume has been deposited in the EMDB (EMDB-8092). The Transcriptome Shotgun Assembly project has been deposited at DDBJ/EMBL/GenBank under the accession GEFJ00000000. The version described in this paper is the first version, GEFJ01000000. RaCI protein sequences have been deposited in GenBank with accession codes KU533794 - KU533797 and BK009406 - BK009408. 
Complement eliminates microbes, modulates the adaptive immune system and plays an important role in the clearance of immune complexes and apoptotic cells, inflammation and tissue regeneration 1,2 .

Antibody complexes (classical pathway, CP), lectin complexes (lectin pathway, LP) or spontaneous hydrolysis of the soluble complement protein $\mathrm{C} 3$ (alternative pathway, AP) initiate a proteolytic cascade that results in the assembly of enzyme complexes (termed $\mathrm{C} 3$ convertases) on surfaces local to the activation stimulus. The $\mathrm{C} 3$ convertases consist of either one molecule of complement $\mathrm{C} 3 \mathrm{~b}$ and the serine protease $\mathrm{Bb}$ (the so-called $\mathrm{C} 3 \mathrm{bBb}$ convertase, generated by $\mathrm{AP}$ ) or one molecule of $\mathrm{C} 4 \mathrm{~b}$ and the serine protease $\mathrm{C} 2 \mathrm{a}$ (the socalled $\mathrm{C} 4 \mathrm{bC} 2 \mathrm{a}$ convertase, generated by $\mathrm{CP}$ and $\mathrm{LP}$ ).

$\mathrm{C} 3$ convertases cleave soluble, circulating $\mathrm{C} 3$, leading to the formation of membranetargeted $\mathrm{C} 3 \mathrm{~b} 3$ and release of the anaphylatoxin C3a fragment 4. Membrane bound C3b further assembles into $\mathrm{C} 3 \mathrm{bBb}$ that amplifies the activation signal. $\mathrm{C} 3 \mathrm{~b}$ and its proteolytic fragments trigger phagocytosis and modulates adaptive immune responses via $\mathrm{B}$ cell stimulation 2. Additional molecules of $\mathrm{C} 3 \mathrm{~b}$ (termed $\mathrm{C} 3 \mathrm{~b}$ ' hereafter) are proposed to associate with the $\mathrm{C} 3$ convertases to form $\mathrm{C} 5$ convertases (either $\mathrm{C} 3 \mathrm{bBbC} 3 \mathrm{~b}$ ' or $\mathrm{C} 4 \mathrm{bC} 2 \mathrm{aC} 3 \mathrm{~b}$ ') when levels of complement activation are high 5, 6, 7, 8, 9. This modulates the activity of the enzyme, which now preferentially cleaves complement component $\mathrm{C} 5$, rather than $\mathrm{C} 3$, to generate $\mathrm{C} 5 \mathrm{~b}$ and the potent anaphylatoxin $\mathrm{C} 5 \mathrm{a}$ that potently attracts and activates neutrophils, monocytes and mast cells 10 . C5b assembles with downstream complement components to generate the membrane attack complex (MAC) that represents the pore forming part of the system responsible for lysing Gram-negative bacteria or inducing tissue inflammation 11. To date the proposed C5 convertase complexes have proved refractory to purification.

Where regulation of complement fails it can turn its destructive capabilities against self and is involved in inflammation, autoimmunity and tissue damage associated with infectious diseases 12, 13. The FDA's approval of administration of an activation-blocking antibody against C5 (Soliris ${ }^{\mathrm{TM}}$; Eculizumab) for patients suffering from paroxysmal nocturnal hemoglobinuria (PNH) or atypical haemolytic uremic syndrome (aHUS) demonstrates the therapeutic utility of blocking the pathway at this point. Inhibition at the level of C5 prevents formation of proinflammatory C5a and MAC but leaves intact generation of the analphylotoxin C3a and cellular opsonisation by C4b and C3b 10,14. Eculizumab is one of the most expensive drugs in the world, costing 500k USD per patient per year (https:// www.nice.org.uk/news/press-and-media/first-nice-highly-specialised-technologies-guidancerecommends-eculizumab-soliris-for-treating-very-rare-life-threatening-blood-disorder). There is therefore much interest in development of novel C5-targeted therapeutics including a molecule (OmCI; coversin), derived from tick-saliva, whose recombinant form is in clinical trials 15 .

A paucity of information about where Eculizumab, OmCI and related drugs bind $\mathrm{C} 5$, as well as a lack of structural insights into the mechanisms underlying inhibition, limits further clinical development. Production of novel C5 inhibitors and structures of C5-inhibtor complexes may therefore lead to development of improved therapies for diseases driven by 
complement. We therefore set out to discover novel C5 inhibitors and determine structures of inhibited C5.

In this study, we identify and characterize a novel protein family of $\sim 8 \mathrm{kDa}$ tick-derived C5 inhibitors, termed the RaCI (Rhipicephalus a ppendiculatus $\mathbf{C} 5$ Inhibitor) family. We present structures that reveal a previously unknown, small $\mathrm{RaCI}$ family protein fold. We determine a series of three structures for ternary complexes of $\mathrm{C} 5$ with two bound inhibitors (OmCI plus three sequence diverse members of the RaCI family). Additionally, we show that Eculizumab binds at a further, unique, site that allows us to assemble and characterize the structure of a quaternary complex C5-OmCI-RaCI-Eculizumab Fab fragment. Our structures reveal that all three classes of inhibitors have distinct binding sites spanning multiple C5 domains. In addition, we design a competition assay that gives results which conflict with previously proposed steric models for OmCI and Eculizumab inhibition. Revisiting the early C5 convertase literature in context of our new data lead us to propose that the C5 convertase components assemble around C5 rather than existing as a pre-assembled entity anchored to the cell surface.

\section{Results}

\section{A novel family of C5 inhibitors}

We studied saliva from the hard tick Rhipicephalus appendiculatus to identify new complement inhibitors that could serve to dissect complement activation and its regulatory pathways, as well as acting as potential pharmaceuticals. A complete salivary gland extract from this species was able to prevent complement-mediated haemolysis (Supplementary Figure 1A). The total protein composition of the complete extract is complex. In order to aid identification of the component responsible for the complement inhibitory activity, the salivary gland extracts (SGE) were sequentially fractionated using anion exchange, reversedphase and size exclusion chromatography. At each stage, the fractions obtained from the chromatographic columns were tested for complement inhibitory activity and fractions with the desired activity were then further fractionated. This eventually generated a fraction highly enriched for inhibition of complement compared to the total extract. This enriched fraction was subjected to LC-MS-MS and expressed sequence tag databases were used to identify peptides 16 . No likely candidate genes were identified. We therefore generated a transcriptome from SGE mRNA using RNAseq. Reanalysis of the LC-MS-MS data against our new transcriptome identified a single candidate gene in the enriched fraction that, when over-expressed in insect cells, showed complement inhibiting activity. We named this protein $\mathrm{RaCI} 1$ ( $\boldsymbol{R}$. appendiculatus Complement Inhibitor 1). This $\sim 8 \mathrm{kDa}$ protein bears no sequence similarity to the previously characterised tick complement inhibitor OmCI $(\sim 17 \mathrm{kDa})$. BLAST searches revealed a second $\mathrm{RaCI}$ homologue in our $R$. appendiculatus transcriptome and five additional homologs in other tick species (hereafter termed RaCI2-7) (Fig. 1A). All RaCI1-7 sequences contain six conserved cysteine residues but display low overall sequence conservation (19\% identity at the protein level; Supplementary Table 1). Supernatants from insect cell lines expressing each of these homologues tested positive for complement inhibition (Supplementary Fig. 1B), demonstrating that RaCI1-7 share a common function. 
We next measured the activity of one of the homologues ( $\mathrm{RaCI} 2$ ) on each of the three major complement activation pathways, using an ELISA-based assay (Wieslab). RaCI homologues were tested for their ability to inhibit all three complement activation pathways (Fig. 1B and Supplementary Fig. 1C) and had $\mathrm{IC}_{50}$ values similar to those previously characterized for the unrelated tick complement inhibitor OmCI. To test whether the RaCI family act upstream or downstream of cleavage of $\mathrm{C} 3$, we assessed whether the anaphylatoxins $\mathrm{C} 3 \mathrm{a}$ and $\mathrm{C} 5 \mathrm{a}$ were formed for each of the three pathways. Our experiments revealed that RaCIs leave C3a levels unaltered but completely abolish formation of C5a in all three pathways (Fig. 1C). We performed a pull-down assay from blood serum to identify the target of RaCI. The pulldown revealed a single protein specifically bound to RaCI. Western blotting using anti-C5 antibodies confirmed that this protein was C5 (Fig. 1D). RaCI thus targets complement after convergence of the three pathways. The higher $\mathrm{IC}_{50}$ in the AP assay is explained by the higher concentration of $\mathrm{C} 5$ present (5.6\% serum in the AP assay versus $1 \%$ serum in $\mathrm{CP}$ and LP pathway assays) rather than by any difference in the ability of RaCIs to inhibit the different pathways. The $\mathrm{IC}_{50} \mathrm{~s}$ calculated correlate with the concentration of $\mathrm{C} 5$ in the assay and reflect an approximately 1:1 molar ratio of inhibitor to $\mathrm{C} 5$. OmCI has been shown to inhibit complement from many different mammalian species 17, 18, therefore we also tested cross-species reactivity for $\mathrm{RaCI}$. $\mathrm{RaCI}$ potently inhibits complement from guinea pig but not from pig, rat, mouse or rabbit. In contrast, as expected, OmCI inhibits complement from all these species (Fig. 1E and Supplementary Fig. 1D) 17, 18. This suggests that the OmCI binding site on $\mathrm{C} 5$ is more highly conserved across species than that targeted by the $\mathrm{RaCI}$ family. Taken together, these data demonstrate that our newly identified RaCI family bind human C5 and block the generation of C5a and MAC, leaving the upstream part of the complement cascade intact.

\section{Crystal structures of C5-inhibitor complexes}

The difference in the ability of $\mathrm{RaCl}$ and $\mathrm{OmCl}$ to inhibit complement across different species, and the lack of sequence similarity between the two inhibitors led us to hypothesize that $\mathrm{RaCl}$ and $\mathrm{OmCl}$ bind different sites on $\mathrm{C} 5$. To purify C5-inhibitor complexes we first used His-tagged $\mathrm{OmCl}$ to isolate $\mathrm{C} 5$ from blood serum (Fig. 1F; Materials and Methods). SEC-MALS data demonstrated that C5 and OmCI bind with a 1:1 molar ratio (Fig. 1G), in agreement with previous data 19 . Adding an excess of free RaCI to the binary complex (followed by further purification) increased the mass of the complex by $\sim 10 \mathrm{kDa}$, corresponding to one copy of $\mathrm{RaCI}$ being incorporated. The formation of a ternary C5OmCl-RaCl complex demonstrates that $\mathrm{RaCI}$ and $\mathrm{OmCI}$ bind different epitopes on $\mathrm{C} 5$ (Fig. $1 \mathrm{~F}, \mathrm{G})$. To gain further insight into the mechanism of inhibition of $\mathrm{C} 5$ cleavage and activation, we wanted to test whether the antibody drug Eculizumab binds another distinct epitope on $\mathrm{C} 5$ to those bound by the tick inhibitors. We generated a custom-made active Fab fragment from the patented sequence for Eculizumab (hereafter termed EcuFab; Supplementary Fig. 2) and added it to the ternary C5-OmCl-RaCl complex (Fig. 2). This resulted in the formation of an $\sim 50 \mathrm{kDa}$ heavier quaternary complex containing $\mathrm{C} 5-\mathrm{OmCl}$ $\mathrm{RaCl}-\mathrm{EcuFab}$ in a 1:1:1:1 ratio demonstrating that all three inhibitors target different binding sites on $\mathrm{C} 5$. 
Crystallization studies were undertaken to gain further insight into the molecular architecture of the inhibited C5 complexes. Crystals for the ternary complex C5-OmCI$\mathrm{RaCI} 3$ were produced that diffracted to $2.6 \AA$ (Table 1; Fig. 2A) and solved by molecular replacement using the existing coordinates for $\mathrm{C} 5$ and $\mathrm{OmCl}$. Phases calculated from this partial model clearly revealed density for the $\mathrm{RaCI}_{3}$ component and this was built de novo. $\mathrm{RaCl} 3$ is wedged between the MG1, MG2 and C5d domains, while OmCI binds a distinct surface of the C5d and CUB domains (see sections below).

Given the low sequence conservation within the RaCI family, we asked whether they all bind C5 in a similar fashion. The answer was provided by crystallographic structures of C5OmCI complexes in the presence of RaCI1 and RaCI2 at resolutions of $3.1 \AA$ and $3.0 \AA$, respectively (Fig. 2B,C). All three RaCI sequences bound the same site on C5. The overall domain arrangement of $\mathrm{C} 5$ is the same as that of the previously reported $\mathrm{C} 5$ structures 19 , with the $\mathrm{C} 345$ c domain adopting a $\mathrm{C} 3$ like orientation, as in the CVF-bound C5 structure 20 (Supplementary Fig. 3,4). The C345c domain has been observed to adopt multiple orientations relative to the main body of $\mathrm{C} 5$ in previous crystallographic structures and SAXS data have suggested that the C345c domain is mobile 19, 20,21. Accordingly, atomic B-factors point to different levels of structural order of C $345 \mathrm{c}$ within our structures. Of note, the other regions that show the greatest variability compared to previous structures are in the C5a and neighboring MG3 domains (Fig 3). In particular, the C-terminus of the anaphylatoxin, which was previously seen 19, 20 in an extended conformation following an unstructured loop, is now completely modelled and adopts a helical conformation as seen in the NMR structure of human C5a (PDB ID:1KJS; 22). This places the Bb cleavage site (Arg751) in the middle of a helix $36 \AA$ distant from the location of the $\mathrm{Bb}$ active site proposed on the basis of earlier models of the $\mathrm{C} 5$ - convertase interaction 20. Furthermore, the cleavage site is completely buried between helices 1 and 4 of the C5a domain and the MG3 domain, showing that significant rearrangement would be required for proteolysis to occur.

\section{The $\mathrm{RaCl}$ - C5 interface}

RaCI binds within the cleft created between the MG1, MG2, and C5d domains (Fig. 2, Supplementary Figs. 5-8). Only the central $\sim 50$ amino acids of the RaCI molecules adopt an ordered fold and are observed in the crystallographic density. The three RaCI molecules are identically folded, consisting of two main loops that are tied together at the base by three conserved disulphide bridges (Fig. 4A). The fold is unique to the RaCI family since no close structural homologues could be identified in the Protein Data Bank, although the fold is somewhat reminiscent of the disulphide-rich structure of small toxins from snake venom 23 . Three stretches of poorly conserved amino acids make extensive contacts with C5 (Fig. 4C), with a number of RaCI-C5 interactions being driven by main chain atoms from RaCI. Extensive use of backbone interactions and a conserved overall fold allows for a high sequence divergence between the RaCI homologues (Fig. 4C).

The relative lack of a canonical hydrophobic core led us to speculate that the $\mathrm{RaCI}$ fold might be inherently flexible. To understand any conformational changes that RaCls undergo upon C5 binding, we solved the solution structure of apo-RaCI2 using NMR spectroscopy 
(Fig. 4B, Table 2). The overlay of the top ten ensemble structures for the apo-RaCI2 revealed the same two ordered loops observed in the structure seen in the context of the C5$\mathrm{RaCI} 2$ complex. However, in the C5-bound structure the tip of loop 1 is bent and loop 2 is twisted compared to the apo structure (Fig. 4B), suggesting that these loops change conformation upon binding of $\mathrm{RaCl} 2$ to $\mathrm{C} 5$. These regions are also characterized by higher intrinsic mobility (as assessed by residual dipolar coupling measurements) for residues that change orientation upon binding (data not shown). As seen in the C5 complex, the N- and Cterminus of RaCI2 are also disordered in the solution structure (Fig. 4B). We made truncated mutants of $\mathrm{RaCI} 2$ to assess whether these poorly conserved and flexible residues are required for inhibition. The ability of the mutants to inhibit both the $\mathrm{CP}$ and $\mathrm{AP}$ were tested (Fig. 4D). Both mutants retained activity with similar potency to the full-length protein, demonstrating that it is the core of RaCI that is required for inhibition of C5 cleavage and activation.

\section{The $\mathrm{OmCl}-\mathrm{C} 5$ interface}

In our crystal structures, OmCI makes contacts with the C5d, CUB and C345c domains of C5 (Fig. 2D, Supplementary Fig. 7-8). Seven amino acids near the $\beta C-\beta D$ loop and at the Cterminus of OmCI make three hydrogen bonds and van der Waals interactions with residues Thr952-Glu958 in the CUB domain. The interactions with the C5d domain are more extensive and include two salt bridges and up to eleven hydrogen bonds. Four discontinuous patches in the OmCI sequence interact with residues between Lys1213 and Asn1241 of the $\mathrm{C} 5 \mathrm{~d}$ domain. Most contacts occur in the $\beta \mathrm{H}$-a 3 region, previously demonstrated to be critical for binding to C5 24. As in the earlier structures of C5 19, 20 portions of the C345c domain are still relatively disordered but a single amino acid from this domain (Phe1631) forms a point of contact with OmCI residues 62 and 63.

OmCI is a bifunctional protein that simultaneously binds C5 and the LTB4 leukotriene 25. Our structures demonstrate the feasibility of simultaneous C5 and LTB4 binding by OmCI (Supplementary Fig. 9). The distinct binding sites are located in opposite faces of $\mathrm{OmCl}$ and the LTB4 binding pocket is accessible in the $\mathrm{C} 5-\mathrm{OmCl}$ complex. As seen in earlier structures of $\mathrm{OmCI} 25,26$, density within the barrel suggests that the C5-bound OmCI pocket is occupied, although the weak density suggests a mixed population or partial occupancy and an atomic model therefore cannot be built.

\section{Structure of the C5-OmCl-RaCl-EcuFab complex}

It is not known where Eculizumab binds C5. Patient data have suggested that Arg885 is positioned in or near the epitope, as Arg885His or Arg885Cys polymorphisms induce drug resistance 27, 28. Antibody - peptide studies have also implicated three short peptides as potentially involved, although these data are hard to interpret due to the fact that two of the peptides identified are buried in the folded structure and the three regions are structurally discontinuous, separated by distances much larger than those that can be incorporated in a single antibody epitope [https://www.google.com/patents/US6355245]. We therefore sought to solve the structure of the $\mathrm{C} 5-\mathrm{OmCl}-\mathrm{RaCl}-\mathrm{EcuFab}$ complex. Attempts to obtain crystals failed. We therefore turned to Transmission Electron Microscopy (TEM) and single-particle analysis 29. 5,587 projection images of different views of the C5-OmCl-RaCl-EcuFab 
complex preserved in negative stain were acquired and an ab initio 3D reconstruction was calculated, without using the X-ray model as a search reference at any point in the analysis. Fitting of the C5-OmCI-RaCI complex structures into the low resolution EM envelope produced a good fit and revealed additional density due to the presence of bound EcuFab. In Figure 5, we present the fit of the $\mathrm{C} 5$ complex to the 3D reconstruction and a comparison of reference-free class averages with projections of a 16- $\AA$-resolution EM density map, simulated from the X-ray structure. The agreement between the projections of the X-ray model and the class averages is excellent and shows additional density due to EcuFab binding the interface between the two lobes of the bipartite $\mathrm{C} 5$ structure in two orthogonal side-views (Fig. 5A,B). The reference-free class averages for similar projection directions point to a moderate flexibility in the positioning of EcuFab relative to the $\mathrm{C} 5$ core (Fig.

5C,D), which may explain why the density due to EcuFab is of slightly lower resolution than the remaining EM density. The EcuFab epitope is centered on the C5 MG7 domain with both Arg885 and the only surface exposed peptide previously implicated as forming part of the epitope (Lys879-Cys-883) lying within the binding site (Fig. 5E).

\section{Inhibition of the $\mathbf{C} 5$ convertases}

The mechanism of conversion of C5 to C5a and C5b by the C5 convertases C3bBbC $3 b$ ' or $\mathrm{C} 4 \mathrm{bC} 2 \mathrm{aC} 3 \mathrm{~b}$ ' is poorly understood, due in part to their very short half-life. Current structural models are based on the highly stable and presumed to be analogous Cobra Venom Factor (CVF)-Bb complex (CVFBb; $\mathrm{CVF}$ is homologous to $\mathrm{C} 3 \mathrm{~b}$ ), which acts in solution without requiring a second $\mathrm{C} 3 \mathrm{~b}$ ' molecule. Current models for the $\mathrm{C} 5$ convertase are based on structures of C3b, C4b, C5, C5-CVF, CVFB and C3bBb 19, 20, 30, 31, 32, 33, and suggest that the MG4, MG5 and MG7 domains of $\mathrm{C} 5$ bind the Bb-bound $\mathrm{C} 3 \mathrm{~b}$ molecule, positioning the active site of the catalytic Bb subunit in the vicinity of the scissile Arg751-Leu752 bond. Neither OmCI nor RaCI bind near the C5a domain, or near the proposed C5 convertasebinding site. Thus inhibition by direct steric hindrance of convertase binding can be ruled out, yet both potently inhibit endogenous convertase-mediated activation of C5 (Fig. 1B; Supplementary Fig. 1C). We tested whether OmCI, RaCI and EcuFab could inhibit CVFBb convertase activity (Fig. 6A). Both RaCI and EcuFab inhibited cleavage by the CVFBb convertase whilst, as previously reported 15 , OmCI did not block CVFBb activity (Fig. 6A). The difference in the ability of the three inhibitors to interfere with a CVF-based convertase contrasts strongly with their equal abilities to inhibit the native $\mathrm{C} 3 \mathrm{bBbC} 3 \mathrm{~b}$ ' and C4bC2aC3b' convertases (Supplementary Figs. 1,2; Fig. 1B).

Next we designed a competition experiment in which highly purified inhibited complexes were added to an ELISA-based CP assay. All binary and ternary complexes of C5 and the tick inhibitors competed with native $\mathrm{C} 5$ demonstrating that they still bind the C5 convertase (Fig. 6B, Supplementary Figure 10). Unlike RaCI and OmCI the monoclonal antibody Eculizumab has been suggested to act sterically by preventing C5 - convertase interactions through binding to the MG7 domain 28, a binding mode now confirmed by our EM data (Supplementary Fig. 11). Surprisingly, complexes of C5 with EcuFab bound (C5-OmCIRaCI-EcuFab, Fig. 6B; C5-EcuFab, Supplementary Fig.5) also inhibited MAC formation in the competition assay suggesting that, unexpectedly, EcuFab does not prevent binding of the inhibited C5 complex to the convertase. Taken together our results demonstrate that C5-CVF 
interactions do not provide a suitable model for interactions between $\mathrm{C} 5$ and the endogenous C5 convertases.

\section{Discussion}

We here demonstrate how three clinically important proteins 34 target C5 revealing why patients carrying an Arg885His or Cys polymorphism cannot be treated with Eculizumab 27 and how OmCI binds LTB4 and C5 simultaneously. Additionally, we identify a new family of $\mathrm{C} 5$ inhibitors that contains highly divergent sequences that nevertheless adopt a conserved fold to target the same site on $\mathrm{C} 5$. $\mathrm{RaCI}$ exhibits cross-species reactivity, potentially enabling study of therapeutic efficacy in animal models of disease, unlike the human-specific Eculizumab. All three inhibitor classes are similarly potent complement inhibitors in vitro, but $\mathrm{RaCI}(\sim 8 \mathrm{kDa})$ is half the size of OmCI $(\sim 17 \mathrm{kDa})$ and much smaller than Eculizumab $(\sim 150 \mathrm{kDa})$, and we show that its size may be further reduced $(\sim 5 \mathrm{kDa})$ to aid drug administration by removal of flexible $\mathrm{N}$ - and $\mathrm{C}$-termini The non-related tick proteins $\mathrm{OmCI}$ and $\mathrm{RaCI}$ target different sites on C5 (C5d-CUB and C5d-MG1-MG2, respectively) and both sites are distinct from that used by Eculizumab. Preliminary data have already demonstrated that OmCI may provide an efficient alternative therapeutic for patients carrying polymorphisms in the Eculizumab binding site (ash.confex.com/ash/2015/webprogram/ Paper79479.html) and it is likely that the RaCI family members will prove equally potent.

It is reasonably well understood how $\mathrm{C} 3$ convertases cleave $\mathrm{C} 3$, but the analogous $\mathrm{C} 5$ convertases remain elusive. $\mathrm{C} 5$ cleavage requires both the $\mathrm{C} 3$ convertase $(\mathrm{C} 4 \mathrm{bC} 2 \mathrm{a}$ or $\mathrm{C} 3 \mathrm{bBb}$ ) and an additional surface-bound molecule of $\mathrm{C} 3 \mathrm{~b}$ ' generated by the $\mathrm{C} 3$ convertase $6,7,8,9,35$. C5 is presumed to bind the endogenous convertase as it binds CVF 20 even though the CVF convertase is not dependent on additional C3b' or surfaces. The exact role of the $\mathrm{C} 3 \mathrm{~b}$ ' molecule in the $\mathrm{C} 5$ convertase activity is disputed. Some studies have shown that C3b' can covalently attach through its $\mathrm{C} 3 \mathrm{~d}$ domain to $\mathrm{C} 4 \mathrm{~b}$ or $\mathrm{C} 3 \mathrm{~b}$ in the $\mathrm{C} 3$ convertase, suggesting that the resulting ternary complexes bind and cleave C5 36, 37 although this has recently been shown not to be required for cleavage 38. Based on crystal structures of $\mathrm{C} 3 \mathrm{bBb}, \mathrm{C} 5-\mathrm{CVF}$ and $\mathrm{C} 4 \mathrm{~b} 20,31,32$ it has been proposed that the reaction of C3b' with the $\mathrm{C} 3$ convertase induces a conformational change in the convertase moiety that leads to a substrate preference for $\mathrm{C} 5$; $\mathrm{C} 5$ binding is presumed to occur on the opposite edge of the $\mathrm{C} 3$ convertase, analogous to the binding of $\mathrm{C} 5$ to $\mathrm{CVF}$, which positions the $\mathrm{Bb}$ or $\mathrm{C} 2 \mathrm{a}$ protease in proximity to the cleavage site (Fig. 7A; 31).

However, our competition assays show that EcuFab-bound complexes still inhibit conversion of C5 despite our structural evidence that the EcuFab-bound C5 would be unable to interact with the convertase in the mode implied by the C5-CVF based convertase models. This implies there must be a distinct interaction event, utilising a different surface to the EcuFab binding site, prior to any C5-CVF-like mode of convertase interaction. This is consistent with an earlier model for the endogenous $\mathrm{C} 5$ convertase, in which $\mathrm{C} 5$ was proposed to directly bind C3b' (coupled to the surface through its thioester), possibly concomitant with a conformational change that enables cleavage by soluble or surface bound $\mathrm{C} 3$ convertase 35 . It would also explain why a high density of $\mathrm{C} 3 \mathrm{~b}$ ' coupled to the surface is required for cleavage by the endogenous convertase 7,38 as you would need two distinct $\mathrm{C} 5$ interaction 
events on the surface (Fig. 7B). Whether C5 remains bound to C3b' while simultaneously binding the $\mathrm{C} 3$ convertase or whether a molecule of $\mathrm{C} 5$ with a short-lived altered conformation transfers to a $\mathrm{C} 3$ convertase remains to be investigated. Both scenarios explain why high densities of surface-coupled C3b' are required.

In addition to lacking a requirement for C3b' and surfaces, the CVF-convertase clearly differs from the endogenous convertase as OmCI, the Staphylococcus aureus inhibitor SSL7 and point mutations in the $\mathrm{C} 345 \mathrm{c}$ domain of $\mathrm{C} 5$ all inhibit endogenous $\mathrm{C} 5$ convertases but not CVF-based convertases (this study, 15, 38). Our new model proposes that cleavage of C5 by endogenous convertases requires two, distinct, interactions at the surface (Fig. 7B). Presumably CVF-convertases do not require the priming interaction to promote productive interactions with the enzyme, perhaps due to higher affinity C5 binding by CVF 39 . Our structures suggest that the EcuFab will sterically prevent interactions with CVF but that $\mathrm{RaCI}$ will not act in the same way (Supplementary Fig. 11). Instead, binding of RaCI may lock C5 into a conformation that cannot interact with either the CVF convertases or the $\mathrm{C} 3$ convertase component of the endogenous pathway. Alternatively, since the C5a scissile bond in our doubly inhibited C5 (Fig. 3C, F) is inaccessible for cleavage by Bb RaCI may act by preventing a structural 'priming' event that rearranges this region to a conformation more similar to that seen in the C5-CVF complex (Fig. 3B, E) 20. It is harder to propose mechanisms for those inhibitors and mutations which differentially effect endogenous and $\mathrm{CVF}$ convertases. However, as for $\mathrm{RaCI}$, they may lock $\mathrm{C} 5$ in a conformation which cannot interact with the endogenous $\mathrm{C} 3$ convertase, but that this lock is not sufficiently strong to prevent interactions with the more tightly binding CVF convertase 39. Although we do not know the nature of the priming event in the endogenous convertase activity we note that the single point of contact of OmCI with the C345c domain of C5 involves a residue (Phe1631), that when mutated, reduces the ability of the endogenous convertase but not the CVF convertase to cleave C5 40. This suggests that the priming event may involve rearrangements of the highly mobile $\mathrm{C} 345 \mathrm{c}$ domain.

In conclusion, our study suggests that the C5 convertase is not a pre-assembled complex. Our data provide strong support for a model in which substantial conformational changes in C5 take place after binding to surface bound C3b', priming C5 for cleavage by the bona fide $\mathrm{C} 3$ convertases. The wide variety of inhibitor structures and binding sites on C5 now revealed suggests that inhibition of this stage of complement activation may be achieved via many mechanistically distinct inhibition strategies potentially allowing fine tuning of therapeutic effects.

\section{Online Methods}

\section{$\mathrm{RaCl}$ purification and identification}

Adult Rhipicephalus appendiculatus ticks were fed on rabbits for three days and glands dissected by Mirko Slovak at the Institute of Zoology (Bratislava, Slovakia). Animal studies were approved by the State Veterinary and Food Administration of the Slovak Republic under decision number $1335 / 15-221$. Dissected glands were stored at $-80{ }^{\circ} \mathrm{C}$ until further use. Approximately 300 glands from males resuspended in PBS were homogenized in a $1 \mathrm{ml}$ dounce homogeniser (Fisher Scientific). Cleared extract was dialyzed against $25 \mathrm{mM} \mathrm{NaPi}$ 
$\mathrm{pH} 7.0$ and loaded onto a MonoQ 5/50 HR column (GE). The pH of the flowthrough was set to $\sim 1$ using $1 \mathrm{M} \mathrm{HCl}$ and protein fractionated on a Dynamax $300 \AA$ A 8 column (Rainin) using $0.1 \%$ Trifluoroacetic acid (buffer A) and $80 \%$ acetonitrile $+0.1 \%$ Trifluoroacetic acid (buffer B). A gradient of 5-80\% buffer B over 20 column volumes (CV) was used. Collected fractions were freeze-dried and resuspended in PBS. Fractions that inhibited complement were pooled and separated on a TSKgel G3000 SW $\mathrm{xl}_{\mathrm{xl}}$ column (Tosoh Bioscience) with PBS as running buffer. The fractions were monitored for activity and the most active was selected for in-solution trypsin digestion followed by LC-MS-MS analysis.

\section{Transcriptome sequencing and assembly}

Fifty salivary glands from males and females were each dissolved in $1 \mathrm{ml}$ Trizol (Ambion). $100 \mu \mathrm{l}$ 1-Bromo-3-chloropropane was added and the sample was vigorously mixed. After centrifugation at $12,000 \mathrm{x}$ g for 15 minutes at $4{ }^{\circ} \mathrm{C}$, the colorless upper phase containing the RNA was transferred to a fresh tube after which an equal volume of $70 \%$ ethanol was added and the sample was vortexed. Total RNA was extracted using the PureLink RNA Mini Kit (Ambion) following the manufacturer's protocol. RNA was eluted in $30 \mu \mathrm{l}$ RNase-free water and the quality of the RNA was assessed on a bio-analyzer (Bio-Rad). The mRNA fraction was selected from approximately $6 \mu \mathrm{g}$ of total RNA using poly-T beads before conversion to cDNA. Second strand cDNA synthesis incorporated dUTP. The cDNA was end-repaired, Atailed and adapter-ligated. Different adapters were used for RNA from male and female ticks. Prior to amplification, samples underwent uridine digestion. Mixed female and male samples were 100bp paired-end sequenced in two lanes on a HiSeq sequencer (Illumina). Sequences were assembled de novo using the Trinity software package with default settings 41. This resulted in 118,614 female and 81,358 male-specific transcripts. From these, six translation frames were generated which were used as a database for LC-MS-MS analysis of purified salivary proteins.

\section{Heterologous protein expression and purification}

His-RaCI and non-tagged homologues were expressed in Drosophila melanogaster S2 suspension cells (Expres2ion Biotechnologies, Denmark). Codon optimized genes (GeneArt, Germany) were cloned into pExpreS2-2 vectors including a BiP signal peptide or the native signal peptide (Supplementary Table 2). Transfections to obtain stable cell lines were carried out following manufacturer's instructions and eight days after transfection the spent medium was tested for complement inhibiting activity.

RaCI family members expressed in insect cells were glycosylated and therefore we expressed RaCI1-4 in Escherichia coli Shuffle T7 cells (New England Biolabs). Codon optimized genes without the signal peptide were cloned into pETM14 vectors (European Molecular Biology Laboratory, Germany), using NcoI or BspHI and NotI restriction sites to yield 3C-cleavable His-tagged proteins (Supplementary Table 2). Cells were grown till $\mathrm{OD}_{600 \mathrm{~nm}}=0.6-0.8$ and induced with $0.5 \mathrm{mM}$ IPTG followed by expression at $21{ }^{\circ} \mathrm{C}$ for 16 hours. Cells were lysed in $150 \mathrm{mM} \mathrm{NaCl}, 50 \mathrm{mM} \mathrm{NaPi} \mathrm{pH} 8.0$ and proteins were purified on HisTrap HP columns (GE). His-tags were removed with HRV 3C protease, followed by a reverse HisTrap column step. RaCIs were subsequently dialysed against $2 \mathrm{mM}$ Cystine, 1 mM Cysteine, $20 \mathrm{mM}$ Ethanolamine $\mathrm{pH} 9.0$, twice for $24-28$ hours at $4{ }^{\circ} \mathrm{C}$ to aid the 
formation of disulfides. RaCI1-3 were then purified on a MonoQ GL column (GE) using 20 $\mathrm{mM}$ Ethanolamine $\mathrm{pH} 9.0$ and a gradient of $\mathrm{NaCl}$ (RaCI1; 0-100 mM, RaCl2; 50-350 mM, RaCI3; 0-250 mM). RaCI4 was purified on a MonoS GL column (GE) using 20mM MES pH 5.5 with a $0-250 \mathrm{mM} \mathrm{NaCl}$ gradient. Protein was dialyzed against PBS and frozen till further use. Truncated RaCI2 variants used in Figure 4D (Supplementary Table 2) were produced as wild type but Cysteine/Cystine dialysis was followed by a Superdex 75 10/30 column (GE) step with PBS as running buffer.

His-tagged OmCI carrying two glycosylation mutations (N78Q/N102Q) (Supplementary Table 2) was expressed using the Kluyveromyces lactis protein expression kit (New England Biolabs) following manufacturer's instructions. The vector was linearized with SacII prior to transformation. Expression was performed in $10 \mathrm{~g} / \mathrm{L}$ yeast extract, $20 \mathrm{~g} / \mathrm{L}$ peptone supplemented with $2 \%$ galactose. After 3 or 4 days of expression spent media was filtered and loaded onto a cOmplete His-Tag Purification Column (Roche) followed by a wash and elution step with PBS supplemented with $250 \mathrm{mM}$ Imidazole. Eluted OmCI was loaded on a Superdex 75 16/60 column (GE), and peak fractions were pooled, dialyzed and frozen until further use.

\section{Purification of C5-complexes}

Out-dated human serum was used for preparation of C5-complexes for crystallization, MALS and some of the competition assays. Serum was thawed and cleared by centrifugation and filtration. $4 \mathrm{mg}$ His-tagged OmCI was incubated with $200 \mathrm{ml}$ serum at room temperature for 30 minutes. C5-OmCI complexes were loaded on 2 x $5 \mathrm{ml} \mathrm{Ni-NTA} \mathrm{columns} \mathrm{(Qiagen).}$ Columns were washed with $30 \mathrm{CV}$ PBS, $20 \mathrm{mM}$ Imidazole followed by elution with $3 \mathrm{CV}$ PBS, $250 \mathrm{mM}$ Imidazole. After dialysis against PBS the complexes were further purified on a MonoQ 10/100 GL column (GE) with 0-35\% gradient of PBS (buffer A) and PBS, 1 M $\mathrm{NaCl}$ (buffer B) over $30 \mathrm{CV}$. Fractions containing pure complexes were dialyzed against 100 $\mathrm{mM} \mathrm{NaCl}, 20 \mathrm{mM}$ HEPES pH 7.5. After dialysis 5-fold molar excess of RaCI was added, samples were incubated for 30 minutes at room temperature and resolved on a Superdex 200 10/30 HR column (GE) with $100 \mathrm{mM} \mathrm{NaCl}, 20 \mathrm{mM}$ HEPES pH 7.5. Ternary complexes were used for crystallization.

For competition experiments, pure C5 (Complement Technologies, USA) was incubated with a 2-fold molar excess of RaCI, OmCI or EcuFab to create the binary complexes.

EcuFab is a custom made Fab fragment using the manufacturer's framework (Ab00296-10.6, Absolute Antibody, UK) and includes the $\mathrm{V}_{\mathrm{L}}$ and $\mathrm{V}_{\mathrm{H}}$ sequences of Eculizumab (European Patent Office: EP0758904 A1). Excess inhibitor was removed with a Superdex 200 5/50 column (GE) in PBS. For MALS and competition experiments, C5OmCI complexes purified from serum were incubated with 3-fold molar excess of RaCI3 and EcuFab. Excess inhibitor was removed by gel filtration on a Superdex 200 10/30 HR column (GE) with $100 \mathrm{mM} \mathrm{NaCl}, 20 \mathrm{mM}$ HEPES pH 7.5. Peak fractions were dialyzed against PBS. 


\section{Haemolysis assay}

Sheep red blood cells (TCS Biosciences) were washed with PBS and sensitized with AntiSheep Red Blood Cell Stroma antibody (cat. no. S1389, Sigma-Aldrich) in $142 \mathrm{mM} \mathrm{NaCl}$, $50 \mathrm{mM}$ Sodium 5,5-diethylbarbiturate, $0.1 \%$ gelatin, $0.15 \mathrm{mM} \mathrm{CaCl}_{2}, 0.5 \mathrm{mM} \mathrm{MgCl}_{2}, \mathrm{pH}$ $7.35\left(\mathrm{GVB}^{2+}\right) .50 \mu \mathrm{l}$ cells $\left(5 \times 10^{8} \mathrm{cells} / \mathrm{ml}\right)$ were mixed with an equal volume of diluted serum in $\mathrm{GVB}^{2+}$ and incubated at $37^{\circ} \mathrm{C}$ for 1 hour while shaking. $100 \mu \mathrm{l}$ of PBS was added and non-lysed cells were pelleted by centrifugation. $100 \mu \mathrm{l}$ of the supernatant was transferred to a clean plate and haemolysis was quantified at $\mathrm{A}_{405 \mathrm{~nm}}$. Cells with $\mathrm{GVB}^{2+}$ instead of serum were used as blank and cells with serum only used for normalization (100\% activity). Mouse haemolysis assays were carried out with fresh serum and $1 \times 10^{8}$ sensitized cells $/ \mathrm{ml}$ in the presence of 5\% MCAR (Complement Technology, USA). Final serum dilutions used were: 1/80 (human), 1/20 (mouse), 1/40 (rabbit), 1/160 (rat), 1/40 (pig) and $1 / 640$ (guinea pig). Human serum was from healthy volunteers, mouse serum was a kind gift from David R. Greaves (University of Oxford, UK), pig serum was a kind gift from Tom E. Mollnes (Oslo University Hospital, Norway), rat and guinea pig serum were from Complement Technology (USA) and rabbit serum was from Pal Freeze (USA).

\section{Complement ELISA}

The effect of RaCI 2 on all three pathways was tested using a Wieslab complement system screen (Euro Diagnostica, Sweden) following manufacturer's instructions. Measurements were subtracted with buffer only and normalised for serum only samples (100\% activity). Other classical and alternative pathway assays were carried out as described by A. Roos et al. 42 , with the following modifications. $2 \mu \mathrm{g} / \mathrm{ml}$ IgM (Bio-Rad) and $1.8 \mathrm{mg} / \mathrm{ml} \mathrm{LPS} \mathrm{from}$ Salmonella typhosa (Sigma-Aldrich) were used to coat ELISA plates. Human serum was diluted 1/100 (classical pathway) or 1/10 (alternative pathway). MAC deposition was detected with primary antibodies against C5b-9 (aE11, Abcam), and secondary anti-mouse IgG HRP conjugate (Promega). HRP activity was detected with 2,2'-Azino-bis(3ethylbenzothiazoline-6-sulfonic acid) liquid substrate (Sigma-Aldrich) and the reaction was stopped by adding an equal volume of $1 \%$ SDS. The absorption was measured at $405 \mathrm{~nm}$. Measurements were subtracted with buffer only samples and normalized for serum only samples (100\% activity). The competition assays were performed using size-exclusion purified inhibited C5 complexes (see purification of C5 complexes above) added to the reaction at the concentrations specified.

\section{C3a and C5a detection}

C3a and C5a levels were determined in supernatants from Wieslab assays in the presence of $0.13 \mu \mathrm{M}$ (classical and lectin pathways) or $0.8 \mu \mathrm{M}$ (alternative pathway) inhibitor, which resulted in maximum inhibition. C3a and C5a levels were detected using MicroVue kits (Quidel) following manufacturer's instructions. For C3a detection, supernatants from Wieslab assays were diluted 40-fold (classical and lectin) and 500-fold (alternative) with diluent. For C5a detection, supernatants from Wieslab assays were diluted 4-fold (classical and lectin) and 20-fold (alternative) with diluent. Background subtraction was done with diluent only samples. 


\section{Pull-down assay}

$\mathrm{RaCI} 2$ and $\mathrm{OmCI}$ at $1 \mathrm{mg} / \mathrm{ml}$ in PBS were coupled to Pierce NHS-Activated magnetic beads (Thermo Scientific) following manufacturer's instructions. $20 \mu \mathrm{l}$ beads were incubated with human serum in the presence of $10 \mathrm{mM}$ EDTA, for 30 minutes at room temperature. Beads were washed three times with $1 \mathrm{ml}$ PBS supplemented with $0.005 \%$ Tween- 20 and once with $100 \mu \mathrm{l}$ PBS. Bound proteins were recovered by boiling the beads in non-reducing SDSPAGE loading buffer, prior to separation on SDS-PAGE gel and analysis by western blot. C5-depleted serum (Complement Technology, USA) was included as a control. Western blots were incubated with 1/2,000 diluted polyclonal anti-C5 antibodies (Calbiochem) followed by $1 / 2,500$ diluted anti-rabbit HRP (Promega). C3 and C4 were detected with antiC3 $(1 / 50,000)$ and anti-C4 $(1 / 150,000)$ polyclonal antibodies (both from Complement Technology, USA) and anti-goat HRP antibodies $(1 / 10,000)$ (Promega). Blots were incubated with ECL substrate (Promega) and imaged with film.

\section{SEC-MALS}

$100 \mu \mathrm{g}(1 \mathrm{mg} / \mathrm{ml})$ of protein was injected onto a Superdex 200 10/30 column (GE Healthcare) and the elution with PBS was monitored using a Dawn Helios II (Wyatt Technology) and an Optilab TrEX (Wyatt Technology). Data were analyzed using ASTRA software (Wyatt Technology).

\section{Cobra venom factor convertase assays}

C5, factor B (fB) and factor D (fD) were purchased from Complement Technology (USA) and Cobra Venom Factor (CVF) from Naja naja kaouthia was purchased from Quidel. CVF, $\mathrm{fB}$ and fD were mixed with a 1:1:0.1 molar ratio in PBS and $5 \mathrm{mM} \mathrm{MgCl} 2$ and incubated for 1 hour at $37^{\circ} \mathrm{C}$. C5 was mixed with a 5-fold molar excess of inhibitor in PBS and incubated for 30 minutes at room temperature. Pre-assembled CVF convertases were added to C5complexes with a $1: 10$ molar ratio, incubated at $37^{\circ} \mathrm{C}$ for 20 hours and separated on a 4-20\% gradient gel (Bio-Rad) in the presence of $10 \mathrm{mM}$ dithiothreitol.

\section{NMR Data Collection and Structure Determination}

All NMR data was collected on Bruker Avance II 500MHz triple resonance, pulse field gradient system equipped with cryoprobe. All experiments were performed on ${ }^{13} \mathrm{C},{ }^{15} \mathrm{~N}$ labelled RaCI2 in PBS (pH7.4). Assignments were performed using both full length (pMJ99) and truncated form (pMJ111) (Table 2). Assignment experiments included CBCANH/CBCA(CO)NH, HNCA/NH(CO)CA, HNCO, HNHA, HBHA(CO)NH, CCCONH and HCCH TOCSY. All data were processed using NMRPipe43 and assigned using Sparky (Goddard \& Kneller, Sparky3, UCSF https://www.cgl.ucsf.edu/home/sparky/). NOE cross-peaks were observed through ${ }^{13} \mathrm{C}$ and ${ }^{15} \mathrm{~N}$ NOESY-HSQC, processed using NMRPipe and peak picked using Sparky. Distance restraints were obtained through a combination of CYANA44 auto assignment and manual assignment. Initial CYANA 44 produced restraints distance restraints were used in CS ROSETTA 45 with the chemical shift data to produce an initial structure for residues Gln34-Leu78, excluding the flexible Nterminal and C-terminal regions. The resulting CSROSETTA44 structure was then used to 
initialise the CYANA44 based structure, using auto assigned and manually assigned NOE based distance restraints, as well as talos+ based angle restraints.

For relaxation experiments all NMR data was collected on Bruker Avance II 600MHz triple resonance, pulse field gradient system equipped with cryoprobe. All experiments were performed on ${ }^{13} \mathrm{C},{ }^{15} \mathrm{~N}$ labelled protein in PBS (pH7.4). $\mathrm{T}_{1}$ and $\mathrm{T}_{2}$ measurements were collected using delays of $(20,125,250,375,500,625,750,875 \mathrm{~ms})$ and $(10,30,50,70,90$, $110,130,150 \mathrm{~ms})$ respectively. Those peaks with exponential decay, baseline resolution and NOE greater than 0.65 were fit to an axially symmetric diffusion tensor and order parameters $\left(S^{2}\right)$ for the residues extracted. Coordinates and restraints have been deposited in the PDB (code 5IEC) and BMRB (code 30024) respectively.

\section{Crystallization, X-ray data collection, Structure determination and refinement}

$4.8 \mathrm{mg} / \mathrm{ml} \mathrm{C5-OmCI-RaCI} 2$ or C5-OmCI-RaCI3 in buffer containing $100 \mathrm{mM} \mathrm{NaCl}, 20 \mathrm{mM}$ HEPES pH 7.5 was mixed with an equal volume of mother liquor containing $10 \%$ PEG 20K, $2 \% \mathrm{v} / \mathrm{v}$ 1,4 Dioxane, $0.1 \mathrm{M}$ Bicine $\mathrm{pH}$ 9.0, and crystallized in $200 \mathrm{nl}$ drops using the vapor diffusion method at $21{ }^{\circ} \mathrm{C}$. Crystals were cryoprotected in mother liquor supplemented with $30 \%$ ethylene glycol and flash frozen in liquid $\mathrm{N}_{2} .5 .5 \mathrm{mg} / \mathrm{ml} \mathrm{C5-OmCI-RaCI1}$ was crystallized as above but mother liquor contained 10\% PEG 6K, 0.1 M Bicine pH 9.0 and crystals were cryoprotected in mother liquor supplemented with 35\% ethylene glycol. Data were collected on beamline I02 at the Diamond Light Source (Harwell, UK) as specified in Table 1. The Structure of C5-OmCI-RaCI3 was solved by molecular replacement using Phaser 46 within CCP4 47 with the structures of C5 (C345c domain removed, PDB ID $3 \mathrm{cu} 7 ; 19)$ and $\mathrm{OmCI}$ (PDB ID 2cm4; 26). The structure of $\mathrm{RaCl} 3$ was manually built into difference density and the model subjected to multiple rounds of manual rebuilding in Coot 48 and refinement in Phenix 49 . Once complete, this structure was used to phase the two other complexes with the sequence of the $\mathrm{RaCI}$ component set to poly alanine prior to molecular replacement, then the sequence rebuilt in the resulting difference density. Following manual rebuilding and refinement (as for the RaCI3 complex), the structures are characterized by the statistics shown in Table 1 with all three having less than $0.1 \%$ Ramachandran outliers and $95 \%$ of residues lying in the favorable regions of the Ramachandran plot. Structure factors and coordinates have been deposited in the PDB with the IDs 5HCC, 5HCD \& 5HCE. Interactions between OmCI and C5, and RaCIs and C5 have been predicted by PISA 50 .

\section{Negative stain specimen preparation, electron microscopy and image processing}

$3 \mu \mathrm{L}$ of quaternary complex C5-OmCI-RaCI-EcuFab solution with a concentration of $\sim 0.1$ $\mathrm{mg} / \mathrm{mL}$ was applied to freshly glow-discharged carbon-coated 400-mesh $\mathrm{Cu}$ grids (Electron Microscopy Sciences). Following incubation for 30 s, excess solution was removed by blotting with a filter paper. The specimen was stained by application of $3 \mu \mathrm{L}$ of $2 \%$ Uranyl Acetate solution. Following a few seconds incubation, excess staining solution was removed by blotting with a filter paper. The staining procedure was repeated twice. CCD images were recorded under low-dose conditions $\left(\sim 20 \mathrm{e}-/ \AA^{2}\right)$ at 0.9-1.3 $\mu$ m underfocus, using a FEI Tecnai T12 transmission electron microscope equipped with a LaB6 filament and operating at $120 \mathrm{kV}$ acceleration voltage. Images were collected at a magnification that gave a 
sampling distance of $2.06 \AA$ at the specimen level. The power spectra of the images were analyzed and images suffering from specimen drift during the exposure were excluded from further analysis. The images were low-pass filtered to the first zero crossing of the contrast transfer function (CTF) before manual windowing of 5,587 single-particle projections, using the particle windowing tool in the EMAN2 suite of programs 51. An ab initio 3D reconstruction was calculated using the PRIME algorithm 52, which is part of the SIMPLE suite of programs 53 , without using the X-ray model as a search reference at any point in the analysis. Fitting the $\mathrm{C} 5$ complex X-ray structures into the low resolution EM envelope validated the $3 \mathrm{D}$ reconstruction. To further assess the validity of the $3 \mathrm{D}$ reconstruction, reference-free 2D class averages were calculated using PRIME50. Next, a 16- $\AA$-resolution EM density map, simulated from the X-ray structure using the pdb2mrc program in EMAN, was used as a search reference to align the reference-free $2 \mathrm{D}$ class averages in $3 \mathrm{D}$. The simulated C5 complex EM density was re-projected in the orientations identified for the class averages in order to demonstrate the excellent agreement with the X-ray model and confirm the presence of the extra density due to bound EcuFab observed in 3D (see Fig. 5, main text). UCSF Chimera was used for automatic real-space rigid body docking 54 . The reconstructed volume has been deposited in the EMDB (accession EMDB-8092)

\section{Supplementary Material}

Refer to Web version on PubMed Central for supplementary material.

\section{Acknowledgements}

We thank N. Darvill, T. Tang \& L. Britton for experimental contributions, S. Jensen for technical support and M. Slovak (Institute of Zoology, Bratislava, Slovakia) for providing salivary glands. We thank the High-Throughput Genomics Group at the Wellcome Trust Centre for Human Genetics (funded by Wellcome Trust grant reference 090532/Z/09/Z and MRC Hub grant G0900747 91070) for the generation of the Sequencing data. This work was financially supported by a Rubicon grant from the Netherlands Organisation for Scientific Research to M.M.J. (825.11.030) and a Wellcome Investigator Award to S.M.L. (100298). We thank the staff at the Clive and Vera Ramaciotti Centre for Structural Cryo-Electron Microscopy at Monash University for assistance with EM image acquisition. We acknowledge Diamond Light Source for time on I02 under proposal MX9306. EM calculations were performed within the Multi-modal Australian ScienceS Imaging and Visualisation Environment (MASSIVE) (www.massive.org.au).

\section{References}

1. Kemper C, Kohl J. Novel roles for complement receptors in T cell regulation and beyond. Mol Immunol. 2013; 56(3):181-190. [PubMed: 23796748]

2. Ricklin D, Hajishengallis G, Yang K, Lambris JD. Complement: a key system for immune surveillance and homeostasis. Nat Immunol. 2010; 11(9):785-797. [PubMed: 20720586]

3. Law SK, Dodds AW. The internal thioester and the covalent binding properties of the complement proteins C3 and C4. Protein Sci. 1997; 6(2):263-274. [PubMed: 9041627]

4. Coulthard LG, Woodruff TM. Is the complement activation product C3a a proinflammatory molecule? Re-evaluating the evidence and the myth. J Immunol. 2015; 194(8):3542-3548. [PubMed: 25848071]

5. Pangburn MK, Rawal N. Structure and function of complement C5 convertase enzymes. Biochem Soc Trans. 2002; 30(Pt 6):1006-1010. [PubMed: 12440962]

6. Rawal N, Pangburn M. Formation of high-affinity C5 convertases of the alternative pathway of complement. J Immunol. 2001; 166(4):2635-2642. [PubMed: 11160326]

7. Rawal N, Pangburn MK. Formation of high affinity C5 convertase of the classical pathway of complement. J Biol Chem. 2003; 278(40):38476-38483. [PubMed: 12878586] 
8. Daha MR, Fearon DT, Austen KF. C3 requirements for formation of alternative pathway C5 convertase. J Immunol. 1976; 117(2):630-634. [PubMed: 950465]

9. Medicus RG, Schreiber RD, Gotze O, Muller-Eberhard HJ. A molecular concept of the properdin pathway. Proc Natl Acad Sci U S A. 1976; 73(2):612-616. [PubMed: 54923]

10. Woodruff TM, Nandakumar KS, Tedesco F. Inhibiting the C5-C5a receptor axis. Mol Immunol. 2011; 48(14):1631-1642. [PubMed: 21549429]

11. Morgan BP. The membrane attack complex as an inflammatory trigger. Immunobiology. 2015

12. Markiewski MM, Lambris JD. The role of complement in inflammatory diseases from behind the scenes into the spotlight. Am J Pathol. 2007; 171(3):715-727. [PubMed: 17640961]

13. Ricklin D, Lambris JD. Complement in immune and inflammatory disorders: pathophysiological mechanisms. J Immunol. 2013; 190(8):3831-3838. [PubMed: 23564577]

14. Mollnes TE, Kirschfink M. Strategies of therapeutic complement inhibition. Mol Immunol. 2006; 43(1-2):107-121. [PubMed: 16011851]

15. Nunn MA, Sharma A, Paesen GC, Adamson S, Lissina O, Willis AC, et al. Complement inhibitor of C5 activation from the soft tick Ornithodoros moubata. J Immunol. 2005; 174(4):2084-2091. [PubMed: 15699138]

16. Nene V, Lee D, Kang'a S, Skilton R, Shah T, de Villiers E, et al. Genes transcribed in the salivary glands of female Rhipicephalus appendiculatus ticks infected with Theileria parva. Insect Biochem Mol Biol. 2004; 34(10):1117-1128. [PubMed: 15475305]

17. Barratt-Due A, Thorgersen EB, Lindstad JK, Pharo A, Lissina O, Lambris JD, et al. Ornithodoros moubata complement inhibitor is an equally effective C5 inhibitor in pigs and humans. J Immunol. 2011; 187(9):4913-4919. [PubMed: 21964028]

18. Hepburn NJ, Chamberlain-Banoub JL, Williams AS, Morgan BP, Harris CL. Prevention of experimental autoimmune myasthenia gravis by rat Crry-Ig: A model agent for long-term complement inhibition in vivo. Mol Immunol. 2008; 45(2):395-405. [PubMed: 17651804]

19. Fredslund F, Laursen NS, Roversi P, Jenner L, Oliveira CL, Pedersen JS, et al. Structure of and influence of a tick complement inhibitor on human complement component 5. Nat Immunol. 2008; 9(7):753-760. [PubMed: 18536718]

20. Laursen NS, Andersen KR, Braren I, Spillner E, Sottrup-Jensen L, Andersen GR. Substrate recognition by complement convertases revealed in the C5-cobra venom factor complex. EMBO J. 2011; 30(3):606-616. [PubMed: 21217642]

21. Laursen NS, Gordon N, Hermans S, Lorenz N, Jackson N, Wines B, et al. Structural basis for inhibition of complement C5 by the SSL7 protein from Staphylococcus aureus. Proc Natl Acad Sci U S A. 2010; 107(8):3681-3686. [PubMed: 20133685]

22. Zhang X, Boyar W, Galakatos N, Gonnella NC. Solution structure of a unique C5a semi-synthetic antagonist: implications in receptor binding. Protein Sci. 1997; 6(1):65-72. [PubMed: 9007977]

23. Kini RM, Doley R. Structure, function and evolution of three-finger toxins: mini proteins with multiple targets. Toxicon. 2010; 56(6):855-867. [PubMed: 20670641]

24. Mans BJ, Ribeiro JM. Function, mechanism and evolution of the moubatin-clade of soft tick lipocalins. Insect Biochem Mol Biol. 2008; 38(9):841-852. [PubMed: 18694828]

25. Roversi P, Ryffel B, Togbe D, Maillet I, Teixeira M, Ahmat N, et al. Bifunctional lipocalin ameliorates murine immune complex-induced acute lung injury. J Biol Chem. 2013; 288(26): 18789-18802. [PubMed: 23625922]

26. Roversi P, Lissina O, Johnson S, Ahmat N, Paesen GC, Ploss K, et al. The structure of OMCI, a novel lipocalin inhibitor of the complement system. J Mol Biol. 2007; 369(3):784-793. [PubMed: 17445829]

27. Nishimura J, Yamamoto M, Hayashi S, Ohyashiki K, Ando K, Brodsky AL, et al. Genetic variants in C5 and poor response to eculizumab. N Engl J Med. 2014; 370(7):632-639. [PubMed: 24521109]

28. Thomas TC, Rollins SA, Rother RP, Giannoni MA, Hartman SL, Elliott EA, et al. Inhibition of complement activity by humanized anti-C5 antibody and single-chain Fv. Mol Immunol. 1996; 33(17-18):1389-1401. [PubMed: 9171898]

29. Elmlund D, Elmlund H. Cryogenic electron microscopy and single-particle analysis. Annu Rev Biochem. 2015; 84:499-517. [PubMed: 25747402] 
30. Janssen BJ, Gomes L, Koning RI, Svergun DI, Koster AJ, Fritzinger DC, et al. Insights into complement convertase formation based on the structure of the factor B-cobra venom factor complex. EMBO J. 2009; 28(16):2469-2478. [PubMed: 19574954]

31. Mortensen S, Kidmose RT, Petersen SV, Szilagyi A, Prohaszka Z, Andersen GR. Structural Basis for the Function of Complement Component C4 within the Classical and Lectin Pathways of Complement. J Immunol. 2015; 194(11):5488-5496. [PubMed: 25911760]

32. Rooijakkers SH, Wu J, Ruyken M, van Domselaar R, Planken KL, Tzekou A, et al. Structural and functional implications of the alternative complement pathway C3 convertase stabilized by a staphylococcal inhibitor. Nat Immunol. 2009; 10(7):721-727. [PubMed: 19503103]

33. Janssen BJ, Christodoulidou A, McCarthy A, Lambris JD, Gros P. Structure of C3b reveals conformational changes that underlie complement activity. Nature. 2006; 444(7116):213-216. [PubMed: 17051160]

34. Reis ES, Mastellos DC, Yancopoulou D, Risitano AM, Ricklin D, Lambris JD. Applying complement therapeutics to rare diseases. Clin Immunol. 2015; 161(2):225-240. [PubMed: 26341313]

35. Vogt W, Schmidt G, Von Buttlar B, Dieminger L. A new function of the activated third component of complement: binding to C5, an essential step for C5 activation. Immunology. 1978; 34(1):2940. [PubMed: 624565]

36. Kinoshita T, Takata Y, Kozono H, Takeda J, Hong KS, Inoue K. C5 convertase of the alternative complement pathway: covalent linkage between two C3b molecules within the trimolecular complex enzyme. J Immunol. 1988; 141(11):3895-3901. [PubMed: 3183384]

37. Takata Y, Kinoshita T, Kozono H, Takeda J, Tanaka E, Hong K, et al. Covalent association of C3b with C4b within C5 convertase of the classical complement pathway. J Exp Med. 1987; 165(6): 1494-1507. [PubMed: 3495629]

38. Berends ET, Gorham RD Jr, Ruyken M, Soppe JA, Orhan H, Aerts PC, et al. Molecular insights into the surface-specific arrangement of complement C5 convertase enzymes. BMC Biol. 2015; 13:93. [PubMed: 26552476]

39. Rawal N, Pangburn MK. Functional role of the noncatalytic subunit of complement C5 convertase. J Immunol. 2000; 164(3):1379-1385. [PubMed: 10640753]

40. Sandoval A, Ai R, Ostresh JM, Ogata RT. Distal recognition site for classical pathway convertase located in the C345C/netrin module of complement component C5. J Immunol. 2000; 165:10661073. [PubMed: 10878385]

41. Grabherr MG, Haas BJ, Yassour M, Levin JZ, Thompson DA, Amit I, et al. Full-length transcriptome assembly from RNA-Seq data without a reference genome. Nat Biotechnol. 2011; 29(7):644-652. [PubMed: 21572440]

42. Roos A, Bouwman LH, Munoz J, Zuiverloon T, Faber-Krol MC, Fallaux-van den Houten FC, et al. Functional characterization of the lectin pathway of complement in human serum. Mol Immunol. 2003; 39(11):655-668. [PubMed: 12493641]

43. Delaglio F, Grzesiek S, Vuister GW, Zhu G, Pfeifer J, Bax A. NMRPipe: a multidimensional spectral processing system based on UNIX pipes. J Biomol NMR. 1995; 6(3):277-293. [PubMed: 8520220]

44. Guntert P. Automated NMR structure calculation with CYANA. Methods Mol Biol. 2004; 278:353-378. [PubMed: 15318003]

45. Tian Y, Opella SJ, Marassi FM. Improved chemical shift prediction by Rosetta conformational sampling. J Biomol NMR. 2012; 54(3):237-243. [PubMed: 23007199]

46. McCoy AJ, Grosse-Kunstleve RW, Adams PD, Winn MD, Storoni LC, Read RJ. Phaser crystallographic software. J Appl Crystallogr. 2007; 40(Pt 4):658-674. [PubMed: 19461840]

47. Winn MD, Ballard CC, Cowtan KD, Dodson EJ, Emsley P, Evans PR, et al. Overview of the CCP4 suite and current developments. Acta Crystallogr D Biol Crystallogr. 2011; 67(Pt 4):235-242. [PubMed: 21460441]

48. Emsley P, Lohkamp B, Scott WG, Cowtan K. Features and development of Coot. Acta Crystallogr D Biol Crystallogr. 2010; 66(Pt 4):486-501. [PubMed: 20383002] 
49. Adams PD, Baker D, Brunger AT, Das R, DiMaio F, Read RJ, et al. Advances, interactions, and future developments in the CNS, Phenix, and Rosetta structural biology software systems. Annu Rev Biophys. 2013; 42:265-287. [PubMed: 23451892]

50. Krissinel E, Henrick K. Inference of macromolecular assemblies from crystalline state. J Mol Biol. 2007; 372:774-797. [PubMed: 17681537]

51. Tang G, Peng L, Baldwin PR, Mann DS, Jiang W, Rees I, et al. EMAN2: an extensible image processing suite for electron microscopy. J Struct Biol. 2007; 157(1):38-46. [PubMed: 16859925]

52. Elmlund H, Elmlund D, Bengio S. PRIME: probabilistic initial 3D model generation for singleparticle cryo-electron microscopy. Structure. 2013; 21(8):1299-1306. [PubMed: 23931142]

53. Elmlund D, Elmlund H. SIMPLE: Software for ab initio reconstruction of heterogeneous singleparticles. J Struct Biol. 2012; 180(3):420-427. [PubMed: 22902564]

54. Yang Z, Lasker K, Schneidman-Duhovny D, Webb B, Huang CC, Pettersen EF, et al. UCSF Chimera, MODELLER, and IMP: an integrated modeling system. J Struct Biol. 2012; 179(3):269_ 278. [PubMed: 21963794] 

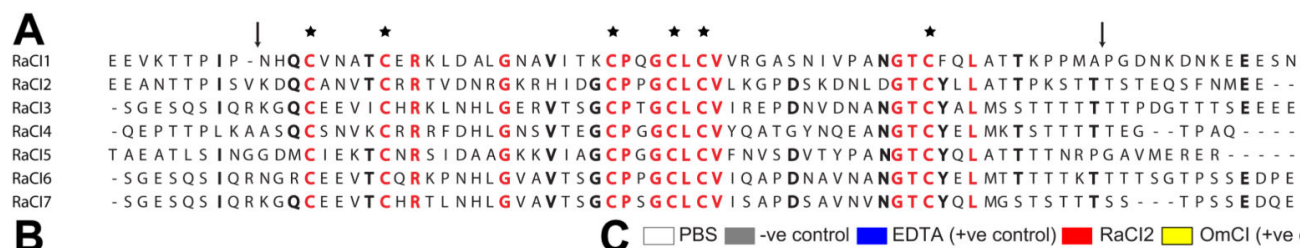

B C $\square$ PBS $\square$-ve control $\square$ EDTA (+ve control) $\square$ RaCl2 $\square$ OmCl (+ve control)

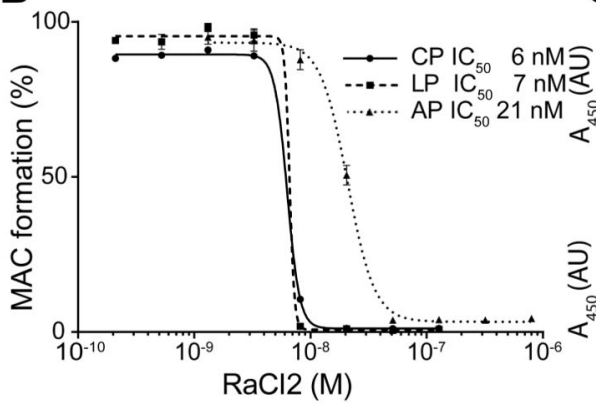

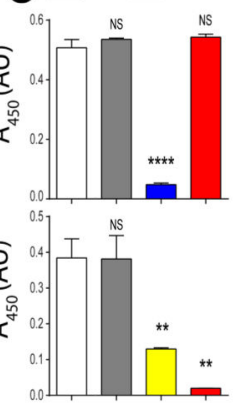

$E$

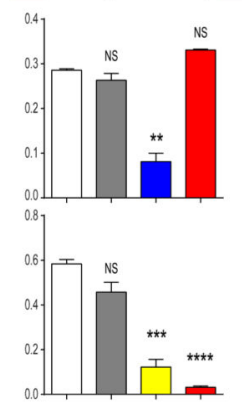

LP

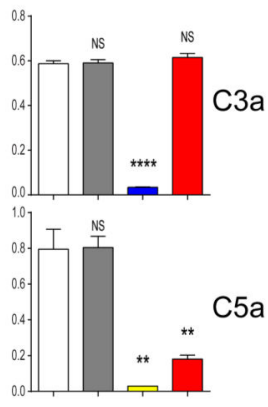

AP
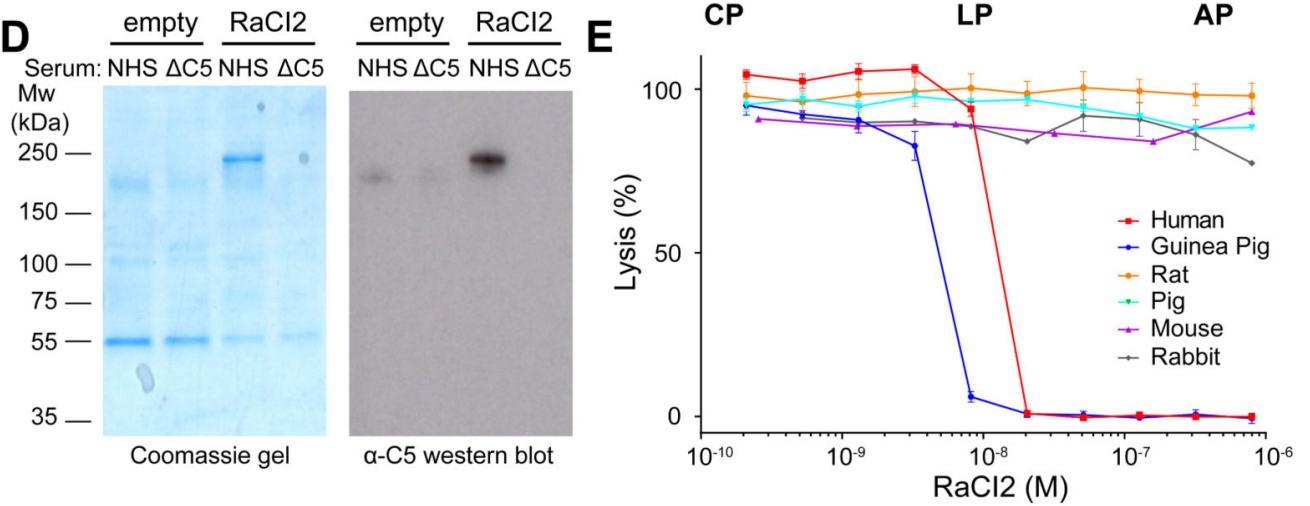

$\mathbf{F}$

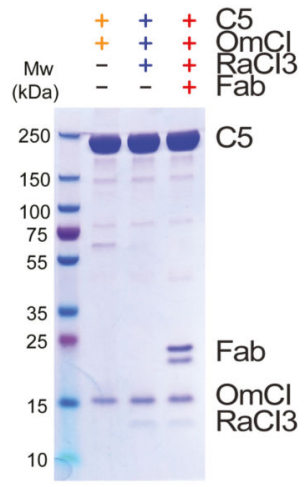

G

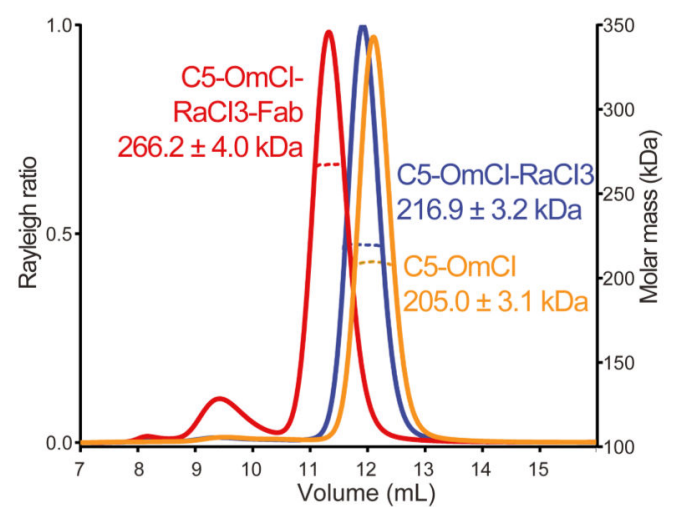

Figure 1. Complement inhibition by RaCI.

(A) Sequence alignment of RaCI family members. RaCI1 is from Rhipicephalus appendiculatus and was purified from salivary gland extract. RaCI5 is a homologue from the $R$. appendiculatus transcriptome described in this study. RaCl2, fusion of the partial sequences in GO496246 and GO496255, Rhipicephalus microplus; RaCI3, EG364159, Dermacentor andersoni; RaCI4, GR908966, Hyalomma marginatum; RaCI6, EG363232, D. andersoni; RaCI7, EG363717, D. andersoni. Residues conserved in five or more RaCIs are in bold and residues conserved in all RaCIs are colored red. Asterisks indicate conserved 
cysteines and arrows indicate truncations used in Figure 4D (B) RaCI2 inhibits classical pathway (CP), lectin pathway (LP) and alternative pathway (AP) in a Wieslab assay. Error bars, s.e.m. ( $n=3$ technical replicates). (C) C3a and C5a levels in supernatants of the Wieslab assay as performed in (B). In the Wieslab samples EDTA treatment (C3a generation) or OmCI (C5a generation) are used as positive controls. The functionally unrelated histamine-binding protein 2, which is structurally related to OmCI, is used as a negative control (C3a \& C5a generation). Error bars, s.e.m. ( $n=3$ Wieslab samples). Significance was calculated using an unpaired two-tailed $t$-test using PBS as a reference. NS $\mathrm{p}>0.05 ; * * \mathrm{p}<0.01 ; * * * \mathrm{p}<0.005 ; * * * * \mathrm{p}<0.001$. (D) Pull-down assay from serum using beads coupled with RaCI2 or empty beads. NHS, normal human serum; $\Delta$ C5, C5-depleted serum. (E) Cross-species activity of RaCI2 in a complement haemolysis assay. Error bars, s.e.m. ( $n=3$ technical replicates). (F) C5 complexes were assembled as described in the methods, purified by size exclusion chromatography and visualized on a Coomassie-stained SDS-PAGE gel and (G) analyzed using SEC-MALS . 

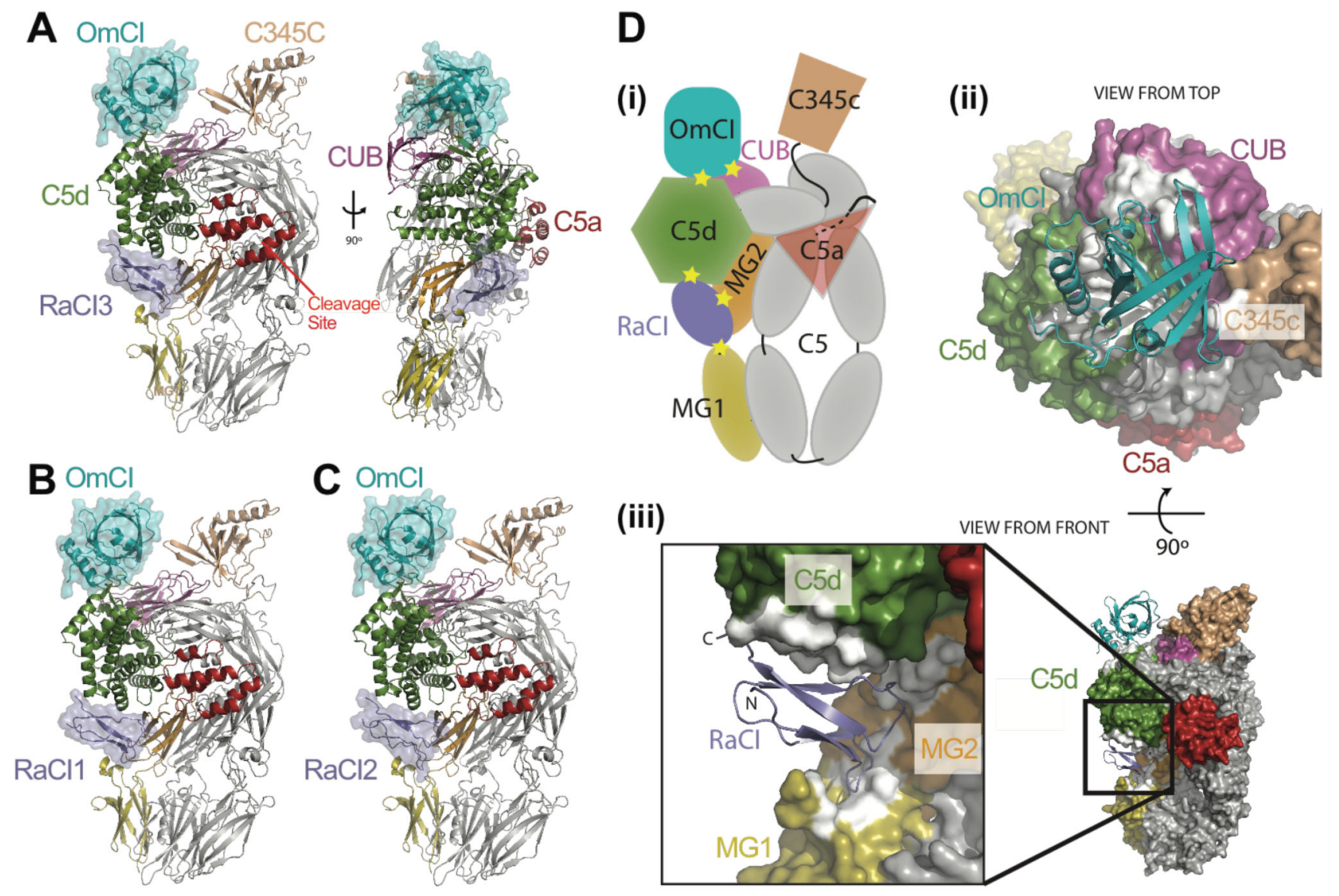

Figure 2. Crystal structures of C5-OmCI-RaCI complexes and binding mode of RaCI family and OmCI inhibitors.

(A) Front and side view of $\mathrm{C} 5$ in complex with $\mathrm{OmCI}$ and $\mathrm{RaCI}_{3}$. Cleavage site, denotes location of cleavage site for release of C5a. Proteins are colored as depicted in panel D(i). All proteins shown as cartoon representations with the two tick inhibitors also shown as semi-transparent surfaces. (B) and (C) crystal structures of C5-OmCI-RaCI1 and C5-OmCI$\mathrm{RaCI} 2$ complexes respectively. (D) Inhibitor binding sites on C5 (i) Cartoon showing domain organization of C5 and interaction sites for both tick-inhibitors (yellow stars). (ii) view onto top of C5 complex with $\mathrm{C} 5$ shown as a surface representation and OmCI as a teal cartoon. (iii) zoom view into the $\mathrm{RaCI}$ binding site. In both (ii) and (iii) the surface of $\mathrm{C} 5$ is colored grey with the exception of the following domains (as in the cartoon shown in (i)); C5a - red, C5d - green, CUB-purple. C5 residues that lie within $4 \AA$ of an inhibitor - white. 

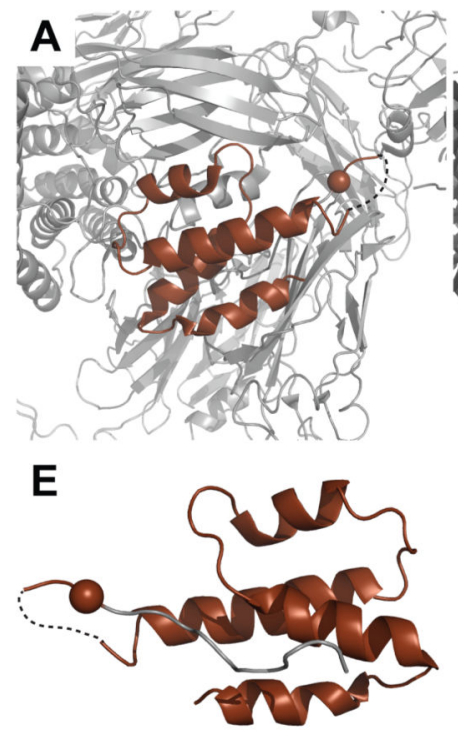

C5 (3CU7)

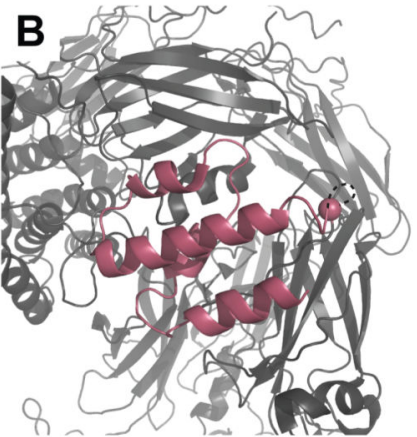

$\mathbf{F}$

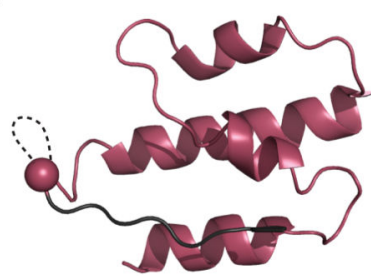

C5-CVF (3PRX)



G

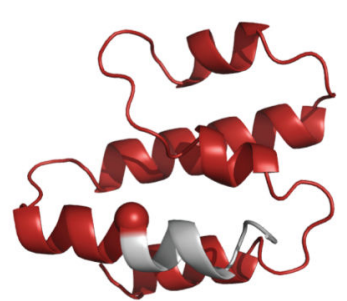

C5-OmCl-RaCl3

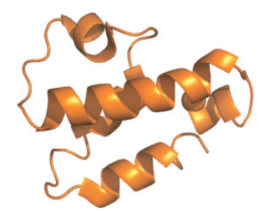

H

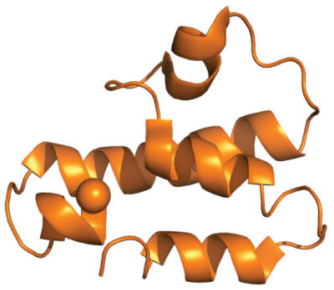

C5a (1KJS)

Figure 3. Remodeling of the C5a domain in the inhibited complex

(A-C) Close-up of the C5a domain (colored) in the C5 structures (grey) as labelled below the panels in the same orientation as the overview shown in Supplementary Figure 4. The peptide that will form the anaphylatoxin after cleavage by $\mathrm{Bb}$ is colored in each picture with the location of the alpha-carbon of Arg751 that will form the C-terminus after cleavage highlighted as a sphere. (D-F) These views are rotated 180 degrees from the top panels, essentially looking from within the body of C5, and show only the residues that will form the anaphylatoxin and an additional ten amino acids C-terminal to the cleavage site (grey). The cleavage site, which adopts an extended conformation following an unstructured loop in the C5 and C5-CVF structures 19, 20, is now seen in the middle of a helix and completely buried between helices 1 and 4 of the C5a domain and MG3. 




Figure 4. The structurally conserved core of the RaCI family members is sufficient for activity. (A) Overlay of RaCI1, $2 \& 3$ in the C5 binding site (colored as in Fig. 3D). The RaCI proteins are shown as alpha carbon traces with the $\mathrm{N}$ - and $\mathrm{C}$-termini and disulphide bonds highlighted. (B) An ensemble of the 10 lowest energy NMR structures of RaCI2 (colored blue to red from $\mathrm{N}$ - to $\mathrm{C}$-termini) shows two conserved loops and highly mobile $\mathrm{N}$ - and $\mathrm{C}$ terminal regions. The ensemble is overlaid on the structure of $\mathrm{RaCI} 2$ (grey) taken from the C5-OmCI-RaCI2 complex. (C) Alignment of RaCI1-3 ordered core residues. Residues that were not seen in the electron density are in lower case. Residues that make van der Waals 
interactions in red, hydrogen bonds in bold and salt bridges are in italics. Brackets indicate conserved disulphides. (D) Elisa-based activation assay (CP - classical pathway, AP alternative pathway) to test inhibitory effect of $\mathrm{RaCI} 2$ mutants that lack N- and C-termini (lacking 10 and 18 amino acids respectively, see Figure 1A for truncation sites) Error bars, s.e.m. ( $n=3$ technical replicates). 

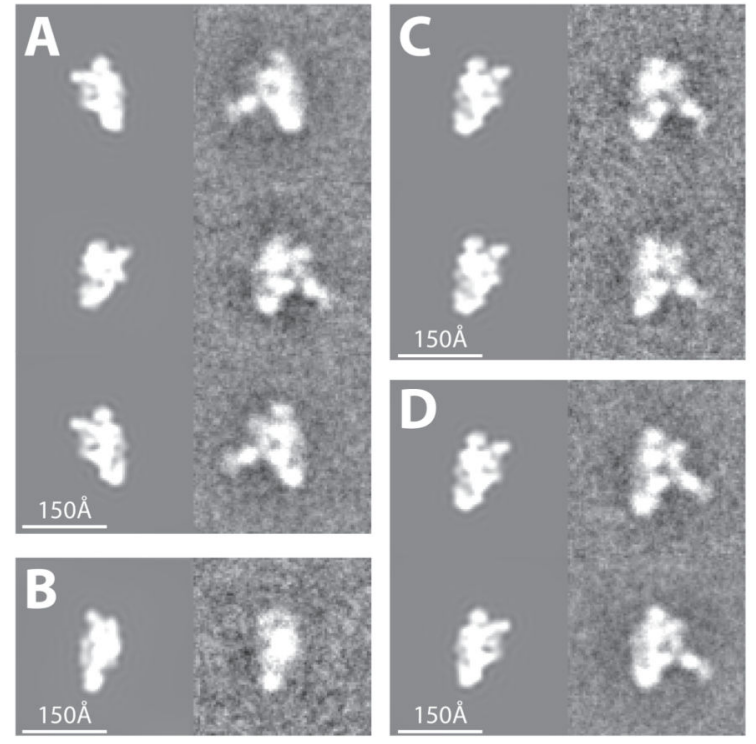

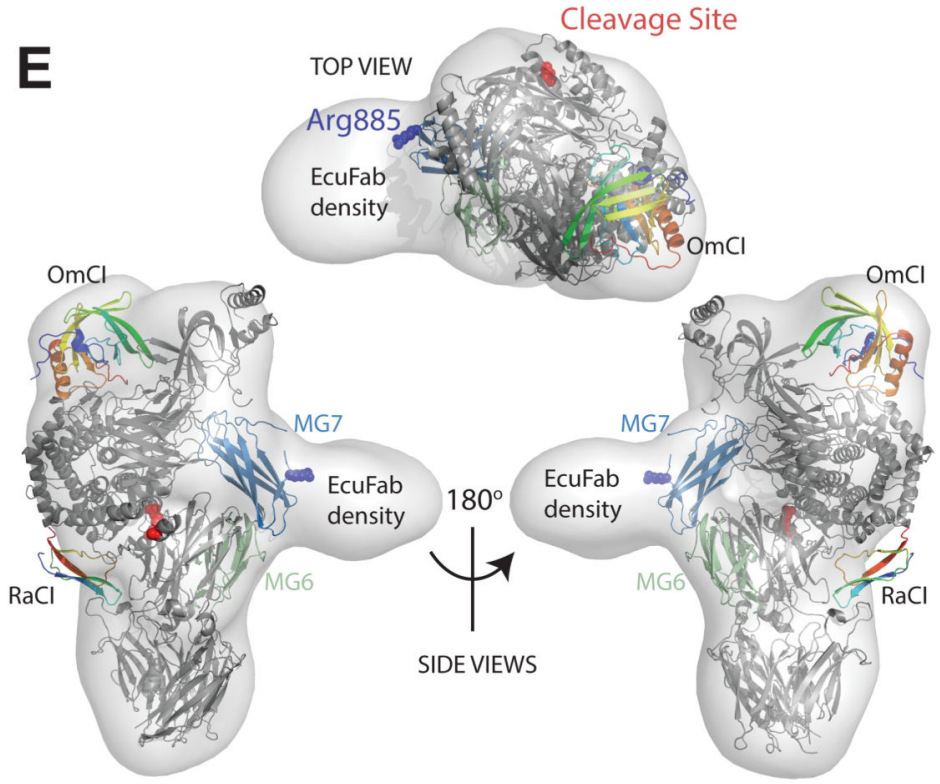

Figure 5. Eculizumab inhibits $\mathrm{C} 5$ activation by binding to a site centered on the MG7 domain. Left panel (A-D): Comparison of projections of a 16-Å-resolution EM density map (left column), simulated from the $\mathrm{C} 5$ complex X-ray structure with reference-free class averages (right column). (A) Side-views related by $\sim 180^{\circ}$ rotation shows the specificity of binding of EcuFab to only one side of the C5 complex. (B) View related by $\sim 90^{\circ}$ rotation with respect to the top and bottom image pairs in (A) reveals, as expected, no additional density due to EcuFab which is not visible in this orientation, but shows good agreement with the X-ray model. (C \& D) Reference-free class averages for similar projection directions point to a moderate flexibility in the positioning of EcuFab relative to the C5 core. (E) Fit of the C5 complex X-ray structure to the low-resolution EM envelope. Top view (top panel) and side views (bottom panel) of the EM-density map (transparent) with the C5 complex X-ray structure docked. Polymorphisms at Arg885 render patients resistant to Eculizumab. The cleavage site is more than $50 \AA$ distant from the EcuFab binding site. 

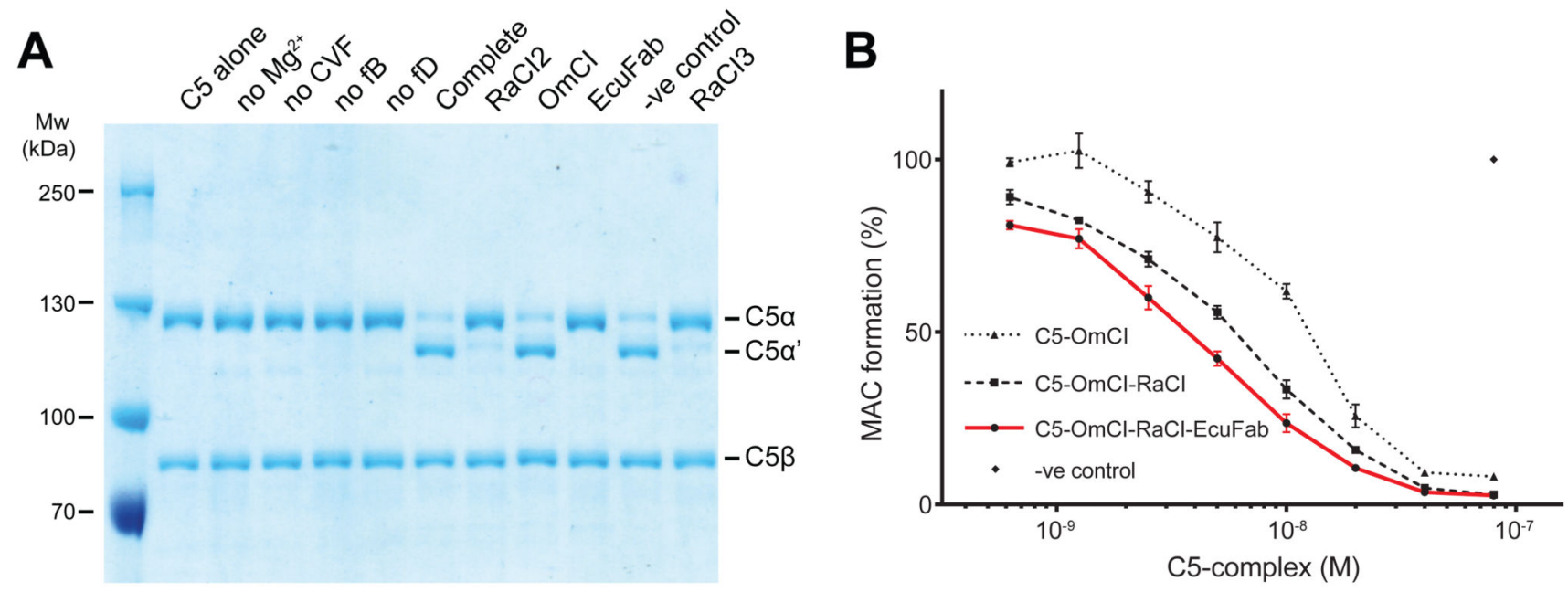

Figure 6. All the inhibited $\mathrm{C} 5$ complexes can themselves inhibit cleavage of $\mathrm{C} 5$ by the native convertase but not all the inhibitors can inhibit cleavage by a CVF convertase.

(A) Coomassie stained gel of $\mathrm{C} 5$ conversion by the CVFBb convertase in the presence of $\mathrm{RaCI}, \mathrm{OmCI}, \mathrm{EcuFab}$ or negative control (histamine-binding protein 2). Lanes 3-6 lack the component indicated; Complete shows the pattern of cleavage when CVFBb is assembled (by incubating $\mathrm{CVF}, \mathrm{fB}$ and $\mathrm{fD}$ ) and given $\mathrm{C} 5$ substrate to act upon in the presence of $\mathrm{Mg}^{2+}$. Final five lanes show the effect of addition of the specified inhibitor or control protein to the C5 substrate prior to incubation with CVFBb. C5: CVF: $\mathrm{fB}$ : fD were present at a final molar ratio of 1: 0.1: 0.1: 0.01. (B) Purified C5-OmCI, C5-OmCI-RaCI and C5-OmCI-RaCI-

EcuFab complexes are added to an Elisa-based classical pathway activation assay to test for competition with native $\mathrm{C} 5$ Error bars, s.e.m. ( $n=3$ technical replicates). 
a

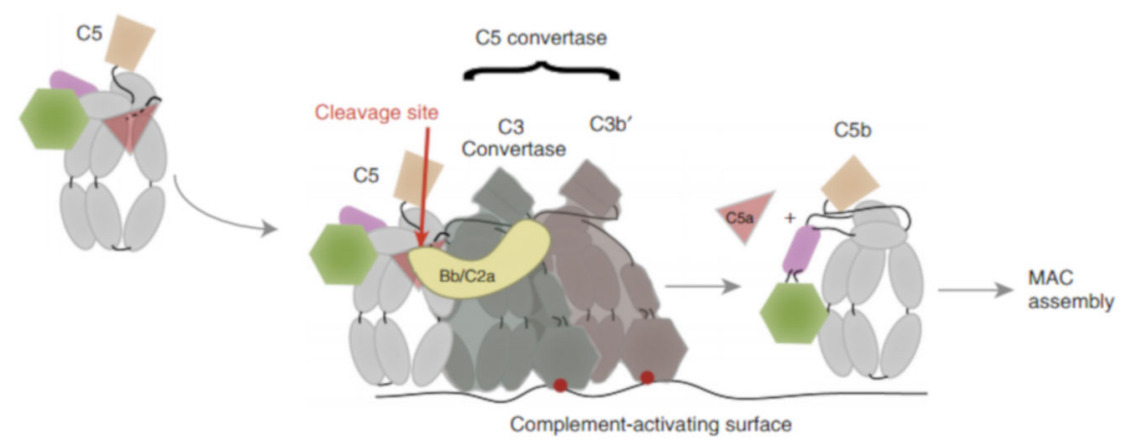

b

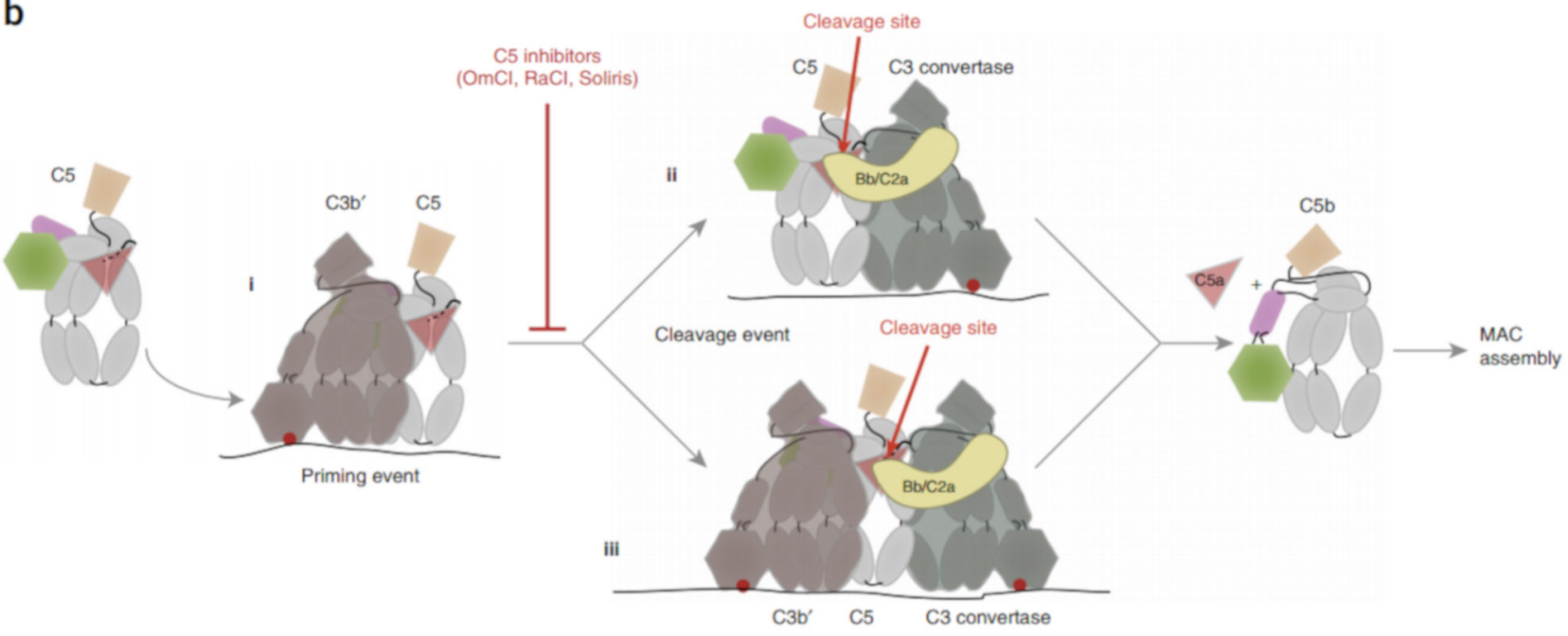

Figure 7. The structures of the inhibited $\mathrm{C} 5$ complexes and competition assay yield an altered view of the $\mathbf{C 5}$ activation pathway.

Cartoons are used to represent key aspects of current and proposed models of C5 activation with the point of covalent attachment to the surface of the $\mathrm{C} 3 \mathrm{~b}$ or $\mathrm{C} 4 \mathrm{~b}$ molecules indicated by the red dot. C3b' - brown; $\mathrm{C} 3$ convertase - grey with enzyme component ( $\mathrm{Bb}$ or $\mathrm{C} 2 \mathrm{a})$ yellow. C5 and C5b colored as in Fig 3. (A) Depiction of current model for activation where a preformed $\mathrm{C} 5$ convertase consisting of $\mathrm{C} 3 \mathrm{BbC} 3 \mathrm{~b}$ ' or $\mathrm{C} 4 \mathrm{bC} 2 \mathrm{aC} 3 \mathrm{~b}$ ' is pre-assembled on the surface and interactions with $\mathrm{C} 5$ are made via the $\mathrm{C} 3$ convertase component. (B) Our data suggest that a first interaction (i) is with the surface bound C3b' molecule and that this either results in conformational changes in $\mathrm{C} 5$ that render it susceptible to cleavage by the $\mathrm{C} 3$ convertases (ii) or that simultaneous interactions by $\mathrm{C} 5$ with the $\mathrm{C} 3 \mathrm{~b}$ ' and the $\mathrm{C} 3$ convertases are required to promote cleavage (iii). 
Table 1

X-ray Data collection and refinement statistics

\begin{tabular}{|c|c|c|c|}
\hline & C5-OmCI-RaCI3 & C5-OmCI-RaCI1 & C5-OmCI-RaCI2 \\
\hline \multicolumn{4}{|l|}{ Data collection } \\
\hline Space group & P 212121 & P 212121 & P 212121 \\
\hline \multicolumn{4}{|l|}{ Cell dimensions } \\
\hline$a, b, c(\AA)$ & $104.9,140.3,211.3$ & $105.3,140.7,210.4$ & $106.0,140.2,209.8$ \\
\hline$a, \beta, \gamma\left({ }^{\circ}\right)$ & $90,90,90$ & $90,90,90$ & $90,90,90$ \\
\hline Resolution ( $(\AA)$ & $66.6-2.6(2.7-2.6)$ & $94.1-3.1(3.3-3.1)$ & $116.6-3.0(3.2-3.0)$ \\
\hline$R_{\text {merge }}$ & $0.08(0.79)$ & $0.12(0.95)$ & $0.11(0.89)$ \\
\hline$I / \sigma I$ & $9.3(1.4)$ & $8.2(1.4)$ & $8.1(1.6)$ \\
\hline Completeness (\%) & $99(100)$ & $100(100)$ & $97(96)$ \\
\hline Redundancy & $3.8(3.7)$ & $4.4(4.3)$ & $4.6(4.5)$ \\
\hline \multicolumn{4}{|l|}{ Refinement } \\
\hline Resolution ( $(\AA)$ & $66.6-2.6(2.7-2.6)$ & $94.1-3.1(3.3-3.1)$ & $116.6-3.0(3.2-3.0)$ \\
\hline No. reflections & $96742(9419)$ & $55783(5464)$ & 64037 (6268) \\
\hline$R_{\text {work }} / R_{\text {free }}$ & $0.231(0.399) / 0.267(0.433)$ & $0.267(0.426) / 0.281(0.413)$ & $0.257(0.405) / 0.282(0.419)$ \\
\hline \multicolumn{4}{|l|}{ No. atoms } \\
\hline Protein & 14432 & 14386 & 14412 \\
\hline Ligand/ion & 68 & 34 & 34 \\
\hline Water & 175 & 0 & 0 \\
\hline \multicolumn{4}{|l|}{$B$ factors } \\
\hline Protein & 74 & 81 & 74 \\
\hline Ligand/ion & 80 & 90 & 79 \\
\hline Water & 51 & - & - \\
\hline \multicolumn{4}{|l|}{ r.m.s. deviations } \\
\hline Bond lengths $(\AA)$ & 0.003 & 0.006 & 0.011 \\
\hline Bond angles $\left({ }^{\circ}\right)$ & 0.61 & 0.75 & 0.89 \\
\hline
\end{tabular}

Number of xtals was 1 for each structure. 


\section{Table 2}

NMR and refinement statistics for RaCI

\begin{tabular}{|c|c|c|}
\hline & Residues 19-88 & Residues 34-78 \\
\hline \multicolumn{3}{|l|}{ NMR distance and dihedral constraints } \\
\hline \multicolumn{3}{|l|}{ Distance constraints } \\
\hline Total NOE & 609 & 436 \\
\hline Intra-residue & 385 & 265 \\
\hline \multicolumn{3}{|l|}{ Inter-reside } \\
\hline Sequential $(|i-\lambda|=1)$ & 140 & 94 \\
\hline Medium-range $(|i-\lambda|<4)$ & 26 & 22 \\
\hline Long-range $(|i-\lambda|>5)$ & 61 & 58 \\
\hline Intermolecular & 0 & 0 \\
\hline Hydrogen bonds & 0 & 0 \\
\hline Total dihedral angle restraints & 62 & 42 \\
\hline \multicolumn{3}{|l|}{ Structure statistics } \\
\hline \multicolumn{3}{|l|}{ Violations (mean \pm s.d.) } \\
\hline Distance constraints $(\AA)$ & $0.21 \pm 0.25$ & $0.22 \pm 0.26$ \\
\hline Dihedral angle constraints $\left(^{\circ}\right)$ & $8.2 \pm 3.8$ & $8.2 \pm 3.8$ \\
\hline Max. dihedral angle violation $\left(^{\circ}\right)$ & 17.3 & 17.3 \\
\hline Max. distance constraint violation $(\AA)$ & 1.58 & 1.58 \\
\hline \multicolumn{3}{|l|}{ Deviations from idealized geometry } \\
\hline Bond lengths $\left({ }^{\circ}\right)$ & 0.001 & 0.001 \\
\hline Bond angles $\left({ }^{\circ}\right)$ & 0.2 & 0.2 \\
\hline Impropers $\left(^{\circ}\right)$ & 0 & 0 \\
\hline \multicolumn{3}{|c|}{ Average pairwise r.m.s. deviation over 10 structures $(\AA)$} \\
\hline Heavy & 4.0 & 0.8 \\
\hline Backbone & 4.3 & 1.4 \\
\hline
\end{tabular}

